Research Article

\title{
Structural Characteristics of Rotate Vector Reducer Free Vibration
}

\author{
Chuan Chen and Yuhu Yang \\ School of Mechanical Engineering, Tianjin University, Tianjin 300072, China \\ Correspondence should be addressed to Chuan Chen; chenchuan1985728@126.com
}

Received 6 January 2017; Revised 9 March 2017; Accepted 30 March 2017; Published 18 April 2017

Academic Editor: Hassan Haddadpour

Copyright (C) 2017 Chuan Chen and Yuhu Yang. This is an open access article distributed under the Creative Commons Attribution License, which permits unrestricted use, distribution, and reproduction in any medium, provided the original work is properly cited.

\begin{abstract}
For RV reducer widely used in robots, vibration significantly affects its performance. A lumped parameter model is developed to investigate free vibration characteristics without and with gyroscopic effects. The dynamic model considers key factors affecting vibration such as involute and cycloid gear mesh stiffness, crankshaft bending stiffness, and bearing stiffness. For both nongyroscopic and gyroscopic systems, free vibrations are examined and compared with each other. Results reveal the specific structure of vibration modes for both systems, which results from symmetry structure of RV reducer. According to vibration of the central components, vibration modes of two systems can be classified into three types, rotational, translational, and planetary component modes. Different from nongyroscopic system, the eigenvalues with gyroscopic effects are complex-valued and speeddependent. The eigenvalue for a range of carrier speeds is obtained by numerical simulation. Divergence and flutter instability is observed at speeds adjacent to critical speeds. Furthermore, the work studies effects of key factors, which include crankshaft eccentricity and the number of pins, on eigenvalues. Finally, experiment is performed to verify the effectiveness of the dynamic model. The research of this paper is helpful for the analysis on free vibration and dynamic design of RV reducer.
\end{abstract}

\section{Introduction}

Rotate Vector (RV) reducer is widely used in the joints of industrial robots due to advantages such as large transmission ratio, high precision, high carrying capacity, high transmission efficiency, and stable working performance. Vibration generated by RV reducer significantly affects the positioning and repeatability of industrial robots, especially for high performance applications.

$\mathrm{RV}$ reducer is a two-stage planetary gear train which joins involute gears and cycloid gears together. The vibration of planetary gears has been extensively studied. Free vibration characteristics of single-stage planetary gears [1-3] and compound planetary gears [4-6] were studied which revealed that unique vibration structure exists in planetary gears. Lin and Parker [7] and Ericson and Parker [8] investigated natural frequency veering of planetary gears. Besides general involute planetary gears, scholars have also researched dynamics of cycloidal planetary gears. Blagojevic et al. [9] and Pascale et al. [10] developed dynamic model of a single stage cycloid drive. Zhang et al. $[11,12]$ formulated a dynamic model of RV reducer which considered mesh stiffness and bearing stiffness. Hsieh [13] studied the dynamic contact and collision conditions of the transmission components in cycloid drives with pinwheel and nonpinwheel designs. The results of [14] showed that structural vibration modes still exist in RV reducer. Gyroscopic effect is neglected in above research of free vibration of planetary gear train.

Gyroscopic effect was first investigated with simple components such as shafts and disks. Researchers have studied the critical speeds and mechanical stability of these gyroscopic systems, which includes axially moving media $[15,16]$, spinning shafts [17], spinning disks [18-20], spinning diskspindle systems [21, 22], and gear-shaft systems [23]. Critical speeds of spinning disks have been experimentally identified in $[18,20,24]$.

In recent years, a few studies have included gyroscopic effect on free vibration of planetary gears. Lin and Parker [25] used eigenvalue derivatives to show the effect of carrier rotation on eigenvalue loci. Abousleiman et al. [26] 


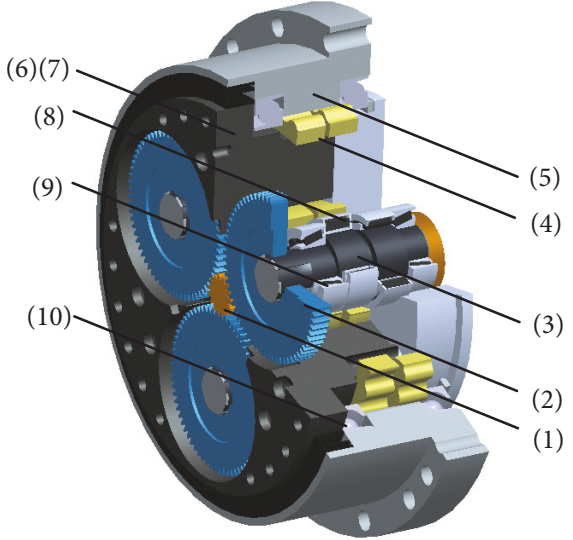

(1) Sun

(2) Planet

(3) Crankshaft

(4) Cycloid gear

(5) Needle wheel

(6) Carrier

(7) Output wheel

(8) Turning arm bearing

(9) Support bearing

(10) Support bearing

(a) CAD model

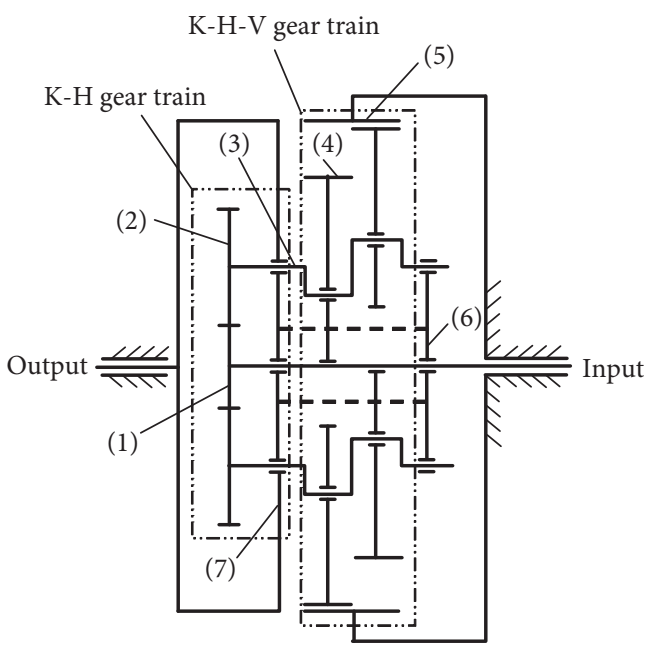
(1) Sun
(2) Planet
(3) Crankshaft
(4) Cycloid gear
(5) Needle wheel
(6) Carrier
(7) Output wheel
(8) Turning arm bearing
(9) Support bearing
(10) Support bearing

FIGURE 1: Schematic of RV reducer.

numerically investigated the effect of carrier rotation on the instantaneous tooth mesh stiffness and dynamic response of planetary gears. They concluded gyroscopic effects should be taken into consideration in high-speed applications. However, the gyroscopic effects are demonstrated by numerical simulation in above articles. Cooley and Parker [27] analytically investigated the modal vibration structure of highspeed planetary gears with gyroscopic effects. Moreover, they also demonstrated unusual gyroscopic system eigenvalue behavior observed in planetary gears [28] and studied the stability of high-speed planetary gears with gyroscopic effects [29].

Despite research above, systematic investigation on free vibration of RV reducer with and without gyroscopic effect has not been considered. The objective of this paper is to explore the structure of natural frequencies and vibration modes and study the effect of key factors on natural frequencies for gyroscopic system. To designers of RV reducer, the prediction of natural frequencies and vibration modes allows them to choose appropriate parameters and avoid resonance conditions when designing this planetary gear system.

\section{Dynamic Model and Equation}

$\mathrm{RV}$ reducer is a two-stage closed planetary gear train, which is characterized by connecting involute gears and cycloid gears as shown in Figure 1. The high-speed stage is a K-H type differential planetary gear train, which consists of sun 1 , planets 2, and the output wheel 7. The low-speed stage is a $\mathrm{K}$ $\mathrm{H}-\mathrm{V}$ type planetary gear train, which consists of crankshafts 3 , cycloid gears 4 , needle wheel 5 , and carrier 6 . The carrier and output wheel are fixed by bolts as one component. A support bearing 9 is installed in the rotation axle of the crankshaft and a turning arm bearing 8 is installed in the junction between the crankshaft and the cycloid gear. A main bearing 10 is installed between the output wheel and the shell of the reducer.

For RV reducer, the planets are of cyclic symmetry. Besides, the crankshafts are in phase, because they are arranged in identical bias direction. The cycloid gears are also in phase due to the arrangement of crankshafts. So RV reducer has special symmetry structure.

A lumped parameter model is shown in Figure 2. Each of the sun, $M$ planets, $M$ crankshafts, $N$ cycloid gears, and the output wheel is treated as rigid bodies. The pins are fixed and the influence on vibration is negligible. Component flexibility, bearings, and gear meshes are represented by linear springs. The supports of the components are modeled as two perpendicular springs with equal stiffness. The transverse stiffness of the sun, planets, crankshafts, cycloid gears, and output wheel is designated as $k_{\mathrm{s}}, k_{\mathrm{a}}, k_{\mathrm{Hb}}, k_{\mathrm{cb}}$, and $k_{\mathrm{o}}$. The torsional stiffness of the sun, planet, and output wheel is represented as $k_{\mathrm{st}}, k_{\mathrm{H}}$, and $k_{\mathrm{ot}}$. The sun-planet and cycloid-pin mesh stiffness is $k_{\mathrm{s} i}(i=1, \ldots, M)$ and $k_{\mathrm{b} j}(j=1, \ldots, N)$.

Each component has three degrees of freedom, two translations and one rotation. Translational coordinates $\widehat{x}_{\mathrm{s}}$, $\widehat{y}_{\mathrm{s}}, \widehat{x}_{\mathrm{o}}$, and $\widehat{y}_{\mathrm{o}}$ are assigned to the sun and output wheel. The hats denote that the matrices and vectors contain dimensional variables. Translational coordinates $\widehat{x}_{\mathrm{p} i}, \widehat{y}_{\mathrm{p} i}, \widehat{x}_{\mathrm{H} i}, \widehat{y}_{\mathrm{H} i}, \widehat{x}_{\mathrm{c} j}$, and $\widehat{y}_{\mathrm{c} j}$ are assigned to planet $i$, crankshaft $i$, and cycloid gear $j$. All rotational coordinates are chosen to be $\theta$. This is illustrated in Figure 2 , where $\widehat{\theta}_{\mathrm{s}}, \widehat{\theta}_{\mathrm{p} i}, \widehat{\theta}_{\mathrm{H} i}, \widehat{\theta}_{\mathrm{c} j}$, and $\widehat{\theta}_{\mathrm{o}}$ are shown.

According to Newton's second law and theorem of angular momentum, the equations of motion can be derived. 


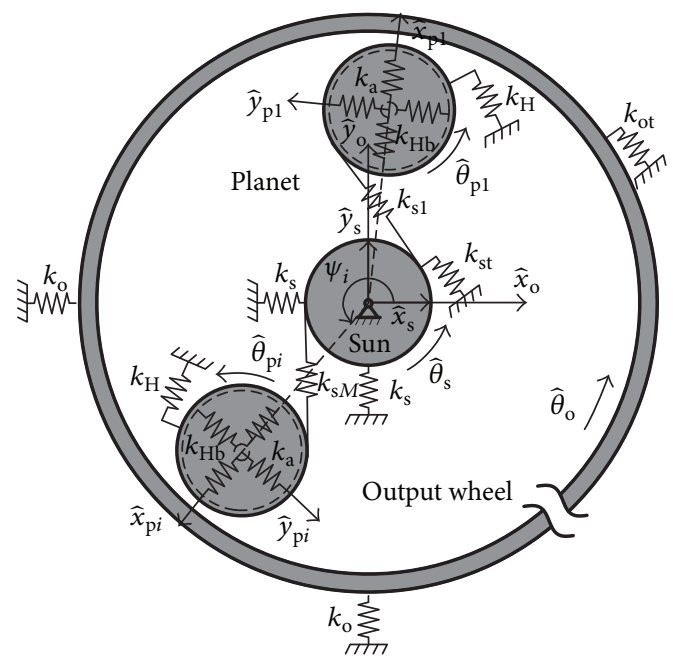

(a) High-speed stage

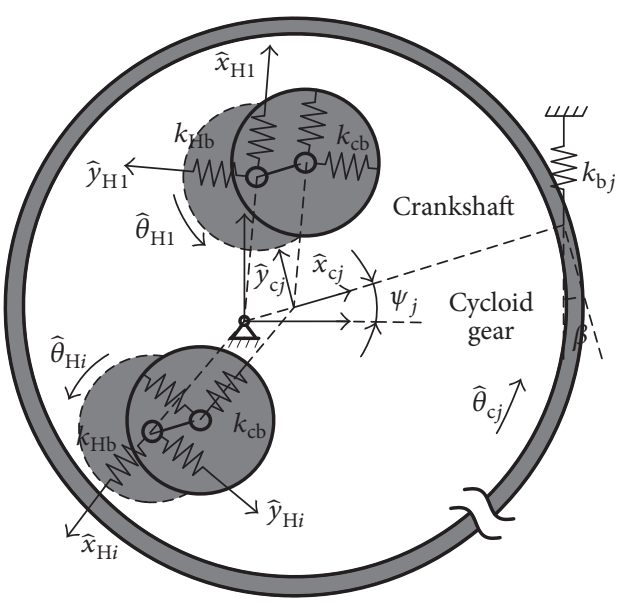

(b) Low-speed stage

FIGURE 2: Lumped parameter model of RV reducer and coordinates.

Taking the cycloid gear as an example, Figure 2(b) shows a cycloid-pin mesh with mass $m_{c j}$, moment of inertia $J_{c j}$, radius of planet distribution circle center $r_{\mathrm{H}}$, and radius of cycloid gear pitch circle $r_{\mathrm{c}}$. Notation $\beta$ is the equivalent pressure angle of the cycloid-pin mesh. The cycloid gear suffers support force from crankshafts and mesh force from pins. The equations are

$$
\begin{aligned}
& m_{\mathrm{c} j}\left(\ddot{\hat{x}}_{\mathrm{c} j}-2 \omega_{\mathrm{o}} \dot{\hat{y}}_{\mathrm{c} j}-\omega_{\mathrm{o}}^{2} \widehat{x}_{\mathrm{c} j}\right) \\
& -\sum_{i=1}^{M} k_{\mathrm{cb}}\left(\delta_{\mathrm{Hicj}} \cos \psi_{\mathrm{H} i}^{\mathrm{cj}}+\delta_{\mathrm{Hicjy}} \sin \psi_{\mathrm{H} i}^{\mathrm{cj}}\right) \\
& +k_{\mathrm{b} j} \delta_{\mathrm{c} j} \sin \beta=0, \\
& m_{\mathrm{c} j}\left(\ddot{\hat{y}}_{\mathrm{c} j}+2 \omega_{\mathrm{o}} \dot{\hat{x}}_{\mathrm{c} j}-\omega_{\mathrm{o}}^{2} \widehat{y}_{\mathrm{c} j}\right) \\
& +\sum_{i=1}^{M} k_{\mathrm{cb}}\left(\delta_{\mathrm{H} i c j x} \sin \psi_{\mathrm{H} i}^{\mathrm{cj}}-\delta_{\mathrm{Hicjy}} \cos \psi_{\mathrm{H} i}^{\mathrm{cj}}\right) \\
& +k_{\mathrm{b} j} \delta_{\mathrm{c} j} \cos \beta=0, \\
& \ddot{\ddot{\hat{\theta}}}_{\mathrm{c} j}-\sum_{i=1}^{M} k_{\mathrm{cb}} \delta_{\mathrm{H} i c j y} r_{\mathrm{H}}+k_{\mathrm{b} j} r_{\mathrm{c}} \delta_{\mathrm{c} j} \cos \beta=0,
\end{aligned}
$$

where $\delta_{\mathrm{Hicj}}$ and $\delta_{\mathrm{Hicj} y}$ are the relative displacement of the $i$ th crankshaft and the $j$ th cycloid gear and $\delta_{\mathrm{c} j}$ is the deflection of the $j$ th cycloid-pin mesh spring. $\delta_{\mathrm{Hicjx}}=\widehat{x}_{\mathrm{H} i}-e \widehat{\theta}_{\mathrm{H} i} \sin \psi_{\mathrm{H} i}^{\mathrm{cj}}-$ $\widehat{x}_{\mathrm{c} j} \cos \psi_{\mathrm{H} i}^{\mathrm{cj}}+\widehat{y}_{\mathrm{c} j} \sin \psi_{\mathrm{H} i}^{\mathrm{cj}}, \delta_{\mathrm{Hicj} y}=\widehat{y}_{\mathrm{H} i}+e \widehat{\theta}_{\mathrm{H} i} \cos \psi_{\mathrm{H} i}^{\mathrm{cj}}-$ $\widehat{x}_{\mathrm{c} j} \sin \psi_{\mathrm{H} i}^{\mathrm{cj}}-\widehat{y}_{\mathrm{c} j} \cos \psi_{\mathrm{H} i}^{\mathrm{cj}}-r_{\mathrm{H}} \widehat{\theta}_{\mathrm{c} j}, \delta_{\mathrm{c} j}=\widehat{x}_{\mathrm{cj}} \sin \beta+\widehat{y}_{\mathrm{cj}} \cos \beta+$ $r_{\mathrm{c}} \widehat{\theta}_{\mathrm{c} j} \cos \beta$. Notation $e$ is crankshaft eccentricity, $\psi_{\mathrm{H} i}^{\mathrm{c} j}=-\pi / 2-$ $2 \pi(i-1) / M+40 \hat{\theta}_{\mathrm{s}} / 185+2 \pi(j-1) / N$ means the angle between the coordinates $\widehat{x}_{\mathrm{H} i}$ and $\widehat{x}_{\mathrm{c} j}$. With resort to the analysis of deflections of cycloid gear support spring, the dynamic model includes crankshaft eccentricity which represents distinction to general planetary gears.
The equations of motion for the sun, planets, crankshafts, and output wheel can be obtained in a similar manner. Assembling the system equations, the governing equations of motion can be written in matrix form as

$$
\widehat{\mathbf{M}} \ddot{\mathbf{q}}+\widehat{\Omega}_{\mathrm{o}} \widehat{\mathbf{G}} \dot{\mathbf{q}}+\left(\widehat{\mathbf{K}}_{\mathrm{b}}+\widehat{\mathbf{K}}_{\mathrm{m}}-\widehat{\Omega}_{\mathrm{o}}^{2} \widehat{\mathbf{K}}_{\Omega}\right) \widehat{\mathbf{q}}=\widehat{\mathbf{F}}(t),
$$

where the matrices $\widehat{\mathbf{M}}, \widehat{\mathbf{G}}, \widehat{\mathbf{K}}_{\mathrm{b}}, \widehat{\mathbf{K}}_{\mathrm{m}}, \widehat{\mathbf{K}}_{\Omega}, \widehat{\mathbf{q}}$, and $\widehat{\mathbf{F}}(t)$ are given in the Appendix of [14].

The influence of external load on free vibration is small and neglected. So the associated free vibration equation is

$$
\widehat{\mathbf{M}} \ddot{\mathbf{q}}+\widehat{\Omega}_{\mathrm{o}} \widehat{\mathbf{G}} \dot{\mathbf{q}}+\left(\widehat{\mathbf{K}}_{\mathrm{b}}+\widehat{\mathbf{K}}_{\mathrm{m}}-\widehat{\Omega}_{\mathrm{o}}^{2} \widehat{\mathbf{K}}_{\Omega}\right) \widehat{\mathbf{q}}=\mathbf{0} .
$$

\section{Natural Frequencies and Vibration Modes without Gyroscopic Effect}

To determine the nature frequencies and vibration modes for nongyroscopic system, the time-invariant system is considered. The gyroscopic effect is neglected. The associated eigenvalue problem of (3) is

$$
\left(\widehat{\mathbf{K}}_{\mathrm{b}}+\widehat{\mathbf{K}}_{\mathrm{m}}-\omega_{n}^{2} \widehat{\mathbf{M}}\right) \widehat{\boldsymbol{\phi}}_{n}=\mathbf{0},
$$

where $\omega_{n}(n=1, \ldots, 6 M+3 N+6)$ are natural frequencies and vibration modes have the form $\widehat{\phi}_{n}=$ $\left[\widehat{\mathbf{p}}_{\mathrm{s}}, \widehat{\mathbf{p}}_{\mathrm{p} 1}, \ldots, \widehat{\mathbf{p}}_{\mathrm{p} M}, \widehat{\mathbf{p}}_{\mathrm{H} 1}, \ldots, \widehat{\mathbf{p}}_{\mathrm{H} M}, \widehat{\mathbf{p}}_{\mathrm{cl}}, \ldots, \widehat{\mathbf{p}}_{\mathrm{cN}}, \widehat{\mathbf{p}}_{\mathrm{o}}\right]^{\mathrm{T}}$ with $\widehat{\mathbf{p}}_{\mathrm{s}}$, $\widehat{\mathbf{p}}_{\mathrm{p} i}, \widehat{\mathbf{p}}_{\mathrm{H} i}, \widehat{\mathbf{p}}_{\mathrm{c} j}$, and $\widehat{\mathbf{p}}_{\mathrm{o}}$ for deflections of the sun, planets, crankshafts, cycloid gears, and the output wheel.

Vibration characteristics are illustrated through a numerical example with the parameters shown in Table 1. The parameters are calculated according to engineering prototype used in experiments.

Suppose there are three planets and cycloid gears. Substitute the parameters in Table 1 into matrices $\widehat{\mathbf{M}}, \widehat{\mathbf{K}}_{\mathrm{b}}$, and $\widehat{\mathbf{K}}_{\mathrm{m}}$. Then the mass and stiffness matrices can be defined and (4) can be solved by numerical simulation. Hence, nature 
TABLE 1: Parameters of an example RV reducer.

\begin{tabular}{|c|c|c|c|c|c|}
\hline Items & Sun & Planet & Crankshaft & Cycloid gear & Carrier \\
\hline Mass/kg & 1.30 & 0.88 & 0.40 & 2.76 & 15.33 \\
\hline Moment of inertia $/ \mathrm{kgm}^{2}$ & $4.44 \times 10^{-4}$ & $1.01 \times 10^{-3}$ & $7.56 \times 10^{-5}$ & $2.09 \times 10^{-2}$ & $1.06 \times 10^{-1}$ \\
\hline Base diameter $/ \mathrm{mm}$ & 10.57 & 48.63 & 2.20 & 85.80 & 63.50 \\
\hline Support stiffness/(N/m) & \multicolumn{5}{|c|}{$k_{\mathrm{s}}=4.19 \times 10^{7} k_{\mathrm{a}}=2.33 \times 10^{8} k_{\mathrm{o}}=1.51 \times 10^{9}$} \\
\hline Bearing stiffness/(N/m) & \multicolumn{5}{|c|}{$k_{\mathrm{Hb}}=9.76 \times 10^{8} k_{\mathrm{cb}}=9.84 \times 10^{8}$} \\
\hline Mesh stiffness/(N/m) & \multicolumn{5}{|c|}{$k_{\mathrm{sn}}=2.68 \times 10^{8} k_{\mathrm{cr}}=8.35 \times 10^{8}$} \\
\hline Torsional stiffness/(Nm/rad) & \multicolumn{5}{|c|}{$k_{\mathrm{st}}=1.16 \times 10^{4} k_{\mathrm{H}}=6.99 \times 10^{4}$} \\
\hline
\end{tabular}

TABLE 2: Natural frequencies $(\mathrm{Hz})$ for various numbers of planets $M$ and $N$ cycloid gears.

\begin{tabular}{|c|c|c|c|c|c|}
\hline$M$ & 3 & 3 & 3 & 4 & 5 \\
\hline$N$ & 3 & 4 & 5 & 3 & 3 \\
\hline \multirow{11}{*}{ Rotational } & 456.56 & 499.63 & 532.20 & 454.13 & 449.47 \\
\hline & 694.49 & 694.04 & 693.79 & 667.28 & 643.89 \\
\hline & 2034.15 & 2025.39 & 2015.27 & 2118.41 & 2181.95 \\
\hline & 2455.49 & 2407.62 & 2349.32 & 2501.34 & 2513.25 \\
\hline & 2645.83 & 2589.33 & 2574.83 & 2932.20 & 3223.88 \\
\hline & 4092.77 & 3979.59 & 3883.12 & 4330.62 & 4470.15 \\
\hline & 5213.45 & 5213.52 & 5213.57 & 5802.57 & 5968.84 \\
\hline & 5583.11 & 5598.21 & 5607.45 & 6017.18 & 6725.28 \\
\hline & 6395.64 & 6578.29 & 6748.81 & 6858.17 & 7382.61 \\
\hline & 16241.42 & 18055.34 & 19703.60 & 16241.42 & 16241.42 \\
\hline & 16576.88 & 18375.22 & 20008.09 & 16688.60 & 16800.30 \\
\hline \multirow{11}{*}{ Translational } & 776.81 & 791.89 & 805.35 & 750.85 & 729.21 \\
\hline & 1999.34 & 2054.02 & 2088.99 & 2057.64 & 2098.51 \\
\hline & 2262.19 & 2302.61 & 2337.30 & 2334.75 & 2372.00 \\
\hline & 2573.12 & 2579.72 & 2585.18 & 2682.82 & 2818.86 \\
\hline & 3713.51 & 3682.54 & 3657.23 & 4203.33 & 4565.57 \\
\hline & 4209.01 & 4128.78 & 4071.80 & 4797.77 & 5304.61 \\
\hline & 4270.38 & 4189.10 & 4132.08 & 4876.53 & 5404.39 \\
\hline & 5255.98 & 5370.58 & 5465.94 & 5405.06 & 5599.51 \\
\hline & 6080.19 & 6126.02 & 6183.41 & 6235.16 & 6402.26 \\
\hline & 16554.35 & 18354.85 & 19989.19 & 16666.35 & 16782.25 \\
\hline & 16585.97 & 18378.10 & 20007.22 & 16707.17 & 16831.56 \\
\hline \multirow{9}{*}{ Planetary component } & & & & 1971.55 & 1971.55 \\
\hline & & & & 2516.17 & 2516.17 \\
\hline & & & & 4808.66 & 4808.66 \\
\hline & & & & 5736.97 & 5736.97 \\
\hline & & & & 16241.37 & 16241.37 \\
\hline & & & & 16241.77 & 16831.10 \\
\hline & & 3877.22 & 3877.22 & & \\
\hline & & 5203.98 & 5203.98 & & \\
\hline & & 5280.81 & 5280.81 & & \\
\hline
\end{tabular}

frequencies can be obtained with $M=3$ and $N=3$. Similarly, natural frequencies can be calculated in other cases. All results are shown in Table 2.

As shown in Table 2, all frequencies are real-valued and structural. Numerical results also reveal the specific structure of vibration modes. Because of the symmetry structure, all vibration modes can be classified into one of three types, rotational modes, translational modes, and planetary component modes. The three vibration modes are defined by the vibration of the central components (the sun and output wheel). They are possible for gyroscopic and nongyroscopic systems. The detailed proofs of the specific structure are presented in [14]. Three vibration modes have the following characteristics. 
Rotational Modes. There are exactly 11 rotational modes, each with an associated natural frequency of multiplicity one. In a rotational mode, the central components have pure rotation and no translation. Planet components (planets, crankshafts, and the cycloid gears) have identical motion. A rotational mode has the form

$$
\widehat{\phi}_{n}=\left[\widehat{\mathbf{p}}_{\mathrm{s}}, \widehat{\mathbf{p}}_{\mathrm{p} 1}, \ldots, \widehat{\mathbf{p}}_{\mathrm{p} 1}, \widehat{\mathbf{p}}_{\mathrm{H} 1}, \ldots, \widehat{\mathbf{p}}_{\mathrm{H} 1}, \widehat{\mathbf{p}}_{\mathrm{c} 1}, \ldots, \widehat{\mathbf{p}}_{\mathrm{cl}}, \widehat{\mathbf{p}}_{\mathrm{o}}\right]^{\mathrm{T}},
$$

where $\widehat{\mathbf{p}}_{\mathrm{s}}=\left[0,0, \widehat{\theta}_{\mathrm{s}}\right]^{\mathrm{T}}, \widehat{\mathbf{p}}_{\mathrm{o}}=\left[0,0, \widehat{\theta}_{\mathrm{o}}\right]^{\mathrm{T}}$.

Translational Modes. There are exactly 11 degenerate pairs of translational modes, where each pair has an associated natural frequency of multiplicity two. All central components have pure translational motion and no rotation. A pair of translational modes has the form

$$
\begin{aligned}
& \widehat{\boldsymbol{\phi}}_{n} \\
& =\left[\widehat{\mathbf{p}}_{\mathrm{s}}, \widehat{\mathbf{p}}_{\mathrm{p} 1}, \ldots, \widehat{\mathbf{p}}_{\mathrm{p} M}, \widehat{\mathbf{p}}_{\mathrm{H} 1}, \ldots, \widehat{\mathbf{p}}_{\mathrm{H} M}, \widehat{\mathbf{p}}_{\mathrm{c} 1}, \ldots, \widehat{\mathbf{p}}_{\mathrm{c} N}, \widehat{\mathbf{p}}_{\mathrm{o}}\right]^{\mathrm{T}}, \\
& \widehat{\boldsymbol{\phi}}_{n}^{\prime} \\
& =\left[\widehat{\mathbf{p}}_{\mathrm{s}}^{\prime}, \widehat{\mathbf{p}}_{\mathrm{p} 1}^{\prime}, \ldots, \widehat{\mathbf{p}}_{\mathrm{p} M}^{\prime}, \widehat{\mathbf{p}}_{\mathrm{H} 1}^{\prime}, \ldots, \widehat{\mathbf{p}}_{\mathrm{H} M}^{\prime}, \widehat{\mathbf{p}}_{\mathrm{c} 1}^{\prime}, \ldots, \widehat{\mathbf{p}}_{\mathrm{c} N}^{\prime}, \widehat{\mathbf{p}}_{\mathrm{o}}^{\prime}\right]^{\mathrm{T}},
\end{aligned}
$$

where $\widehat{\mathbf{p}}_{\mathrm{s}}=\left[\widehat{x}_{\mathrm{s}}, \widehat{y}_{\mathrm{s}}, 0\right]^{\mathrm{T}}, \widehat{\mathbf{p}}_{\mathrm{o}}=\left[\widehat{x}_{\mathrm{o}}, \hat{y}_{\mathrm{o}}, 0\right]^{\mathrm{T}}$.

Planetary Component Modes. There are exactly 6 or 3 degenerate pairs of modes, each with an associated natural frequency of multiplicity $M-3$ or $N-3$. The central components have no motion and the planet components deflect. A planetary component mode has the form

$$
\begin{aligned}
& \widehat{\boldsymbol{\phi}}_{n}=\left[\mathbf{0}, \widehat{\mathbf{p}}_{\mathrm{p} 1}, \ldots, \widehat{\mathbf{p}}_{\mathrm{p} M}, \widehat{\mathbf{p}}_{\mathrm{H} 1}, \widehat{\mathbf{p}}_{\mathrm{H} M}, \mathbf{0}, \mathbf{0}, \ldots, \mathbf{0}\right]^{\mathrm{T}}, \\
& \widehat{\boldsymbol{\phi}}_{n}=\left[\mathbf{0}, \mathbf{0}, \ldots, \mathbf{0}, \widehat{\mathbf{p}}_{\mathrm{c} 1}, \ldots, \widehat{\mathbf{p}}_{\mathrm{cN}}, \mathbf{0}\right]^{\mathrm{T}} .
\end{aligned}
$$

When the number of crankshaft or cycloid gear is 2, the rotational and translational modes still exist. However, the multiplicity of natural frequency associated with translational mode is one. So only the first three orders of natural frequency with $M=3$ and $N=2$ are listed in Table 5 .

\section{Natural Frequencies and Vibration Modes with Gyroscopic Effect}

4.1. Typical Vibration Modes. Equation (3) is nondimensionalized by introducing the variables

$$
\begin{aligned}
& x_{\mathrm{s}}=\frac{\widehat{x}_{\mathrm{s}}}{r_{\mathrm{p}}}, \\
& y_{\mathrm{s}}=\frac{\widehat{y}_{\mathrm{s}}}{r_{\mathrm{p}}}, \\
& \theta_{\mathrm{s}}=\frac{\widehat{\theta}_{\mathrm{s}}}{\theta_{\mathrm{p}}},
\end{aligned}
$$

$$
\begin{aligned}
& x_{\mathrm{o}}=\frac{\widehat{x}_{\mathrm{o}}}{r_{\mathrm{p}}}, \\
& y_{\mathrm{o}}=\frac{\widehat{y}_{\mathrm{o}}}{r_{\mathrm{p}}}, \\
& \theta_{\mathrm{o}}=\frac{\widehat{\theta}_{\mathrm{o}}}{\theta_{\mathrm{p}}}, \\
& \gamma_{\mathrm{s}}=\frac{r_{\mathrm{s}}}{r_{\mathrm{p}}}, \\
& \gamma_{\mathrm{p}}=\frac{r_{\mathrm{p}}}{r_{\mathrm{p}}}, \\
& \gamma_{\mathrm{H}}=\frac{r_{\mathrm{H}}}{r_{\mathrm{p}}}, \\
& \gamma_{\mathrm{c}}=\frac{r_{\mathrm{c}}}{r_{\mathrm{p}}} \text {, } \\
& \varepsilon=\frac{e}{r_{\mathrm{p}}}, \\
& \mu_{\mathrm{s}}=\frac{m_{\mathrm{s}}}{m_{\mathrm{p}}}, \\
& v_{\mathrm{s}}=\frac{J_{\mathrm{s}}}{m_{\mathrm{p}}}, \\
& \mu_{\mathrm{p} i}=\frac{m_{\mathrm{p} i}}{m_{\mathrm{p}}}, \\
& v_{\mathrm{p} i}=\frac{J_{\mathrm{p} i}}{m_{\mathrm{p}}}, \\
& \mu_{\mathrm{H} i}=\frac{m_{\mathrm{H} i}}{m_{\mathrm{p}}}, \\
& v_{\mathrm{H} i}=\frac{J_{\mathrm{H} i}}{m_{\mathrm{p}}} \text {, } \\
& \mu_{\mathrm{c} j}=\frac{m_{\mathrm{c} j}}{m_{\mathrm{p}}}, \\
& v_{\mathrm{c} j}=\frac{J_{\mathrm{c} j}}{m_{\mathrm{p}}}, \\
& \mu_{\mathrm{o}}=\frac{m_{\mathrm{o}}}{m_{\mathrm{p}}} \text {, } \\
& v_{\mathrm{o}}=\frac{J_{\mathrm{o}}}{m_{\mathrm{p}}}, \\
& \kappa_{s i}=\frac{k_{\mathrm{si}}}{k_{\mathrm{a}}}, \\
& \kappa_{\mathrm{b} j}=\frac{k_{\mathrm{b} j}}{k_{\mathrm{a}}}, \\
& \kappa_{\mathrm{s}}=\frac{k_{\mathrm{s}}}{k_{\mathrm{a}}} \text {, }
\end{aligned}
$$




$$
\begin{gathered}
\kappa_{\mathrm{a}}=\frac{k_{\mathrm{a}}}{k_{\mathrm{a}}} \\
\kappa_{\mathrm{cb}}=\frac{k_{\mathrm{cb}}}{k_{\mathrm{a}}} \\
\kappa_{\mathrm{Hb}}=\frac{k_{\mathrm{Hb}}}{k_{\mathrm{a}}}, \\
\kappa_{\mathrm{H}}=\frac{k_{\mathrm{H}}}{k_{\mathrm{a}}} \\
\kappa_{\mathrm{st}}=\frac{k_{\mathrm{st}}}{k_{\mathrm{a}}}
\end{gathered}
$$

where $i=1,2, \ldots, M$ and $j=1,2, \ldots, N$.

Using (8) in (3) gives

$$
\mathbf{M} \ddot{\mathbf{q}}+\Omega_{\mathrm{o}} \mathbf{G} \dot{\mathbf{q}}+\left(\mathbf{K}_{\mathrm{b}}+\mathbf{K}_{\mathrm{m}}-\Omega_{\mathrm{o}}^{2} \mathbf{K}_{\Omega}\right) \mathbf{q}=\mathbf{0},
$$

where the matrices $\mathbf{M}, \mathbf{G}, \mathbf{K}_{\mathrm{b}}, \mathbf{K}_{\mathrm{m}}, \mathbf{K}_{\Omega}$, and $\mathbf{q}$ are given in Appendix.

Substitution of the separable solution $\mathbf{q}=\phi e^{\lambda t}$ into the homogeneous form of (9) gives the polynomial eigenvalue problem

$$
\lambda^{2} \mathbf{M} \phi+\lambda \Omega_{\mathrm{o}} \mathbf{G} \phi+\left(\mathbf{K}_{\mathrm{b}}+\mathbf{K}_{\mathrm{m}}-\Omega_{\mathrm{o}}^{2} \mathbf{K}_{\Omega}\right) \boldsymbol{\phi}=\mathbf{0},
$$

where $\phi=\left[\mathbf{p}_{\mathrm{s}}, \mathbf{p}_{\mathrm{p} 1}, \ldots, \mathbf{p}_{\mathrm{p} M}, \mathbf{p}_{\mathrm{H} 1}, \ldots, \mathbf{p}_{\mathrm{H} M}, \mathbf{p}_{\mathrm{c} 1}, \ldots, \mathbf{p}_{\mathrm{c} N}, \mathbf{p}_{\mathrm{o}}\right]^{\mathrm{T}}$ is the eigenvector with gyroscopic effect and $\mathbf{p}_{\mathrm{s}}, \mathbf{p}_{\mathrm{p} i}, \mathbf{p}_{\mathrm{H} i}, \mathbf{p}_{\mathrm{c} j}$, and $\mathbf{p}_{\mathrm{o}}$ are deflections of the sun, planets, crankshafts, cycloid gears, and the output wheel with gyroscopic effect.

Equation (10) is cast into the state space form as follows:

$$
\mathbf{A} \dot{\mathbf{u}}+\mathbf{B u}=\mathbf{0},
$$

where $\mathbf{u}=[\dot{\mathbf{q}}, \mathbf{q}]^{\mathrm{T}} \cdot \mathbf{A}=\left[\begin{array}{cc}\mathbf{M} & \mathbf{0} \\ \mathbf{0} & \mathbf{K}\end{array}\right]$ and $\mathbf{B}=\left[\begin{array}{cc}\Omega_{\mathrm{o}} \mathbf{G} & \mathbf{K} \\ -\mathbf{K} & \mathbf{0}\end{array}\right]$ are the state matrix operators.

Substitution of the separable solution $\mathbf{u}=\chi e^{\lambda t}$ into (11) gives the gyroscopic state eigenvalue problem:

$$
\lambda \mathbf{A} \chi+\mathbf{B} \chi=\mathbf{0}
$$

where $\chi=[\lambda \Phi, \Phi]^{\mathrm{T}}$.

The eigenvalues can be calculated by solving (12) with parameters in Table 1. The eigenvalues are complex-valued and natural frequencies are defined as the imaginary part. Natural frequencies and their multiplicities with different number of crankshafts and cycloid gears are shown in Table 3. Numerical results of vibration modes reveal that all vibration modes for RV reducers can be classified into one of three types. The symmetry structure of RV reducer leads to structural characteristics of vibration modes.

Figure 3 shows representative modes shapes for RV reducer with four planets and three cycloid gears. The following paragraphs summarize the modal characteristics obtained from numerical results.

Rotational Modes. There are exactly eleven natural frequencies, each associated with rotational mode of multiplicity

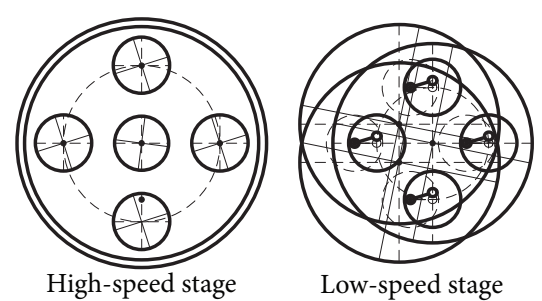

(a) Gyroscopic rotational mode

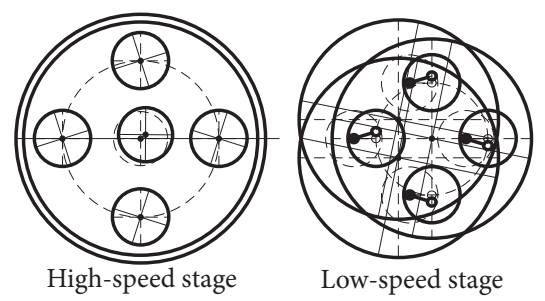

(b) Gyroscopic translational mode
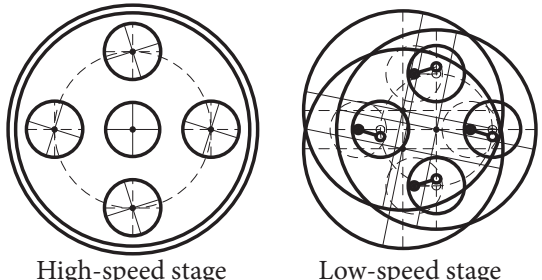

(c) Gyroscopic planetary component mode

FIGURE 3: Vibration modes with gyroscopic effect.

one. In a rotational mode, all central components have pure rotation and no translation and all planetary components (the planets, the crankshafts, and the cycloid gears) have identical motion. These characteristics are consistent with nongyroscopic system. In nongyroscopic systems, the eigenvectors are real and the motions are either in-phase (where two motions have the same sign) or 180 deg out of phase (where two motions have opposite sign). However, the eigenvectors of gyroscopic systems are complex which physically means a phase difference exists between the motions of different coordinates. Hence, there are eleven pairs of complex conjugate rotational modes of gyroscopic systems. Although the motions of each planetary component are still identical and in-phase with every other planetary component, the motions of different coordinates for each planet are not in-phase with each other. A gyroscopic rotational mode has the form

$$
\begin{aligned}
& \boldsymbol{\phi}_{n} \\
& \quad=\left[\mathbf{p}_{\mathrm{s}}, \mathbf{p}_{\mathrm{p} 1}, \ldots, \mathbf{p}_{\mathrm{p} 1}, \mathbf{p}_{\mathrm{H} 1}, \ldots, \mathbf{p}_{\mathrm{H} 1}, \mathbf{p}_{\mathrm{c} 1}, \ldots, \mathbf{p}_{\mathrm{c} 1}, \mathbf{p}_{\mathrm{o}}\right]^{\mathrm{T}} .
\end{aligned}
$$

Translational Modes. For nongyroscopic system, there are eleven pairs of real eigensolutions, where each pair has an associated natural frequency of multiplicity two. All central components of translational modes have pure translational motion and no rotational motion. For gyroscopic system, there are exactly twenty-two pairs of complex conjugate translational modes. The translational mode eigenvalues are 
TABLE 3: Nondimensional natural frequencies $(\mathrm{Hz})$ and $\operatorname{Im}(\lambda)$, for $\mathrm{RV}$ reducer.

\begin{tabular}{|c|c|c|c|c|c|c|}
\hline \multirow{2}{*}{ Mode type } & \multicolumn{2}{|c|}{$M=3 N=3$} & \multicolumn{2}{|c|}{$M=3 N=4$} & \multicolumn{2}{|c|}{$M=4 N=3$} \\
\hline & $\omega_{\mathrm{o}}=0$ & $\omega_{\mathrm{o}}=0.2$ & $\omega_{\mathrm{o}}=0$ & $\omega_{\mathrm{o}}=0.2$ & $\omega_{\mathrm{o}}=0$ & $\omega_{\mathrm{o}}=0.2$ \\
\hline \multirow{11}{*}{ Rotational } & $0.1762(1)$ & $0.1664(1)$ & $0.1928(1)$ & $0.1848(1)$ & $0.1752(1)$ & $0.1621(1)$ \\
\hline & $0.2680(1)$ & $0.2474(1)$ & $0.2678(1)$ & $0.2384(1)$ & $0.2575(1)$ & $0.2421(1)$ \\
\hline & $0.7849(1)$ & $0.7638(1)$ & $0.7815(1)$ & $0.7580(1)$ & $0.8174(1)$ & $0.7995(1)$ \\
\hline & $0.9475(1)$ & $0.9286(1)$ & $0.9290(1)$ & $0.9081(1)$ & $0.9652(1)$ & $0.9453(1)$ \\
\hline & $1.0209(1)$ & $1.0036(1)$ & $0.9991(1)$ & $0.9783(1)$ & $1.1314(1)$ & $1.1249(1)$ \\
\hline & $1.5793(1)$ & $1.2686(1)$ & $1.5356(1)$ & $1.2107(1)$ & $1.6710(1)$ & $1.4730(1)$ \\
\hline & $2.0117(1)$ & $1.5775(1)$ & $2.0117(1)$ & $1.5774(1)$ & $2.2390(1)$ & $1.9574(1)$ \\
\hline & $2.1543(1)$ & $2.0981(1)$ & $2.1602(1)$ & $2.1196(1)$ & $2.3218(1)$ & $2.1979(1)$ \\
\hline & $2.4679(1)$ & $2.3849(1)$ & $2.5383(1)$ & $2.4495(1)$ & $2.6463(1)$ & $2.5018(1)$ \\
\hline & $6.2670(1)$ & $6.2638(1)$ & $6.9669(1)$ & $6.9641(1)$ & $6.2670(1)$ & $6.2638(1)$ \\
\hline & $6.3964(1)$ & $6.3934(1)$ & $7.0904(1)$ & $7.0876(1)$ & $6.4396(1)$ & $6.4366(1)$ \\
\hline \multirow{22}{*}{ Translational } & \multirow{2}{*}{$0.2997(2)$} & $0.1551(1)$ & \multirow{2}{*}{$0.3056(2)$} & $0.1605(1)$ & \multirow{2}{*}{$0.2897(2)$} & 0.1568 (1) \\
\hline & & $0.2724(1)$ & & $0.3116(1)$ & & 0.3789 (1) \\
\hline & \multirow{2}{*}{$0.6176(2)$} & $0.3995(1)$ & \multirow{2}{*}{$0.6564(2)$} & $0.4081(1)$ & \multirow{2}{*}{$0.6940(2)$} & $0.4265(1)$ \\
\hline & & $0.5102(1)$ & & $0.5349(1)$ & & $0.6122(1)$ \\
\hline & \multirow{2}{*}{$0.8286(2)$} & $0.7522(1)$ & \multirow{2}{*}{$0.8335(2)$} & $0.7539(1)$ & \multirow{2}{*}{$0.8542(2)$} & $0.7884(1)$ \\
\hline & & 0.7993 (1) & & $0.7960(1)$ & & $0.8532(1)$ \\
\hline & \multirow{2}{*}{$0.9634(2)$} & $0.8781(1)$ & \multirow{2}{*}{$0.9672(2)$} & $0.8698(1)$ & \multirow{2}{*}{$0.9782(2)$} & 0.9275 (1) \\
\hline & & $0.9529(1)$ & & $0.9479(1)$ & & $1.0043(1)$ \\
\hline & \multirow{2}{*}{$1.4328(2)$} & $1.1043(1)$ & \multirow{2}{*}{1.4209 (2) } & $1.0623(1)$ & \multirow{2}{*}{$1.6219(2)$} & $1.4003(1)$ \\
\hline & & 1.1187 (1) & & $1.0828(1)$ & & $1.4042(1)$ \\
\hline & \multirow{2}{*}{$1.6219(2)$} & 1.1515 (1) & \multirow{2}{*}{$1.5903(2)$} & $1.1126(1)$ & \multirow{2}{*}{$1.8506(2)$} & $1.4515(1)$ \\
\hline & & $1.2382(1)$ & & $1.2069(1)$ & & $1.5204(1)$ \\
\hline & \multirow{2}{*}{$1.6430(2)$} & $1.4459(1)$ & $16121(2)$ & $1.4345(1)$ & $18760(2)$ & $1.6329(1)$ \\
\hline & & $1.4472(1)$ & & $1.4358(1)$ & & $1.6359(1)$ \\
\hline & $20281(2)$ & $2.0083(1)$ & $20723(2)$ & $2.0490(1)$ & $20856(2)$ & $2.0666(1)$ \\
\hline & & 2.0449 (1) & & $2.0929(1)$ & & $2.1002(1)$ \\
\hline & $23461(2)$ & $2.3207(1)$ & $23638(2)$ & $2.3424(1)$ & 24059 (2) & $2.3695(1)$ \\
\hline & & $2.3762(1)$ & & $2.3903(1)$ & & $2.4487(1)$ \\
\hline & $6.3877(2)$ & $6.3800(1)$ & $70825(2)$ & 7.0761 (1) & $6.4310(2)$ & $6.4216(1)$ \\
\hline & & $6.3800(1)$ & & 7.0761 (1) & & $6.4216(1)$ \\
\hline & $6.3998(2)$ & $6.3915(1)$ & $70914(2)$ & $7.0846(1)$ & $6.4466(2)$ & $6.4364(1)$ \\
\hline & & $6.3930(1)$ & & $7.0856(1)$ & $0.4460(2)$ & $6.4384(1)$ \\
\hline & & & $1.4961(1)$ & 14881 (1) & $0.7608(1)$ & $0.7467(1)$ \\
\hline & & & & & 0.9709 (1) & $0.9501(1)$ \\
\hline Planetary component & & & $2.0080(1)$ & $15723(1)$ & $1.8555(1)$ & $1.8516(1)$ \\
\hline & & & & $1.5 / 23(1)$ & $2.2137(1)$ & $2.2127(1)$ \\
\hline & & & $2.0377(1)$ & $1.6174(1)$ & $6.2670(1)$ & $6.2638(1)$ \\
\hline & & & & $1.01 / 4(1)$ & $6.2671(1)$ & $6.2639(1)$ \\
\hline
\end{tabular}

degenerate when the speed of carrier is zero and split for nonzero speed. As the speed of carrier increases, one purely imaginary eigenvalue increases and the other one decreases. The translational motions of different coordinates for the central components are 90 deg out of phase. Unlike cyclic symmetry system, the motions of planet have phase relationship with a phase-shift. The motion of planetary components of RV reducer does not exist in general phase

$$
\begin{aligned}
\phi_{n} & =\left[\mathbf{p}_{\mathrm{s}}, \mathbf{p}_{\mathrm{p} 1}, \ldots, \mathbf{p}_{\mathrm{p} M}, \mathbf{p}_{\mathrm{H} 1}, \ldots, \mathbf{p}_{\mathrm{H} M}, \mathbf{p}_{\mathrm{c} 1}, \ldots, \mathbf{p}_{\mathrm{cN}}, \mathbf{p}_{\mathrm{o}}\right]^{\mathrm{T}}, \\
\boldsymbol{\phi}_{n}^{\prime} & =\left[\mathbf{p}_{\mathrm{s}}^{\prime}, \mathbf{p}_{\mathrm{p} 1}^{\prime}, \ldots, \mathbf{p}_{\mathrm{p} M}^{\prime}, \mathbf{p}_{\mathrm{H} 1}^{\prime}, \ldots, \mathbf{p}_{\mathrm{H} M}^{\prime}, \mathbf{p}_{\mathrm{c} 1}^{\prime}, \ldots, \mathbf{p}_{\mathrm{cN}}^{\prime}, \mathbf{p}_{\mathrm{o}}^{\prime}\right]^{\mathrm{T}} .
\end{aligned}
$$

Planetary Component Modes. Planetary component modes occur in systems with four or more planetary components.
For nongyroscopic system, there are three or six real eigensolutions, where each has an associated natural frequency of multiplicity $M-3$ or $N-3$. The central components have no motion. The motions of any planetary components are scalar multiples of the motions of the first planetary component. For gyroscopic system, there are three or six pairs of complex conjugate eigensolutions. Although the motions of each planetary component are not in-phase with each other, the motions of each planetary component are in-phase or $180 \mathrm{deg}$ out of phase with every other planetary component.

$$
\begin{aligned}
& \boldsymbol{\phi}_{n}=\left[\mathbf{0}, \mathbf{p}_{\mathrm{p} 1}, \ldots, \mathbf{p}_{\mathrm{p} M}, \mathbf{p}_{\mathrm{H} 1}, \ldots, \mathbf{p}_{\mathrm{H} M}, \mathbf{0}, \mathbf{0}, \ldots, \mathbf{0}\right]^{\mathrm{T}}, \\
& \boldsymbol{\phi}_{n}=\left[\mathbf{0}, \mathbf{0}, \ldots, \mathbf{0}, \mathbf{p}_{\mathrm{c} 1}, \ldots, \mathbf{p}_{\mathrm{cN}}, \mathbf{0}\right]^{\mathrm{T}}
\end{aligned}
$$




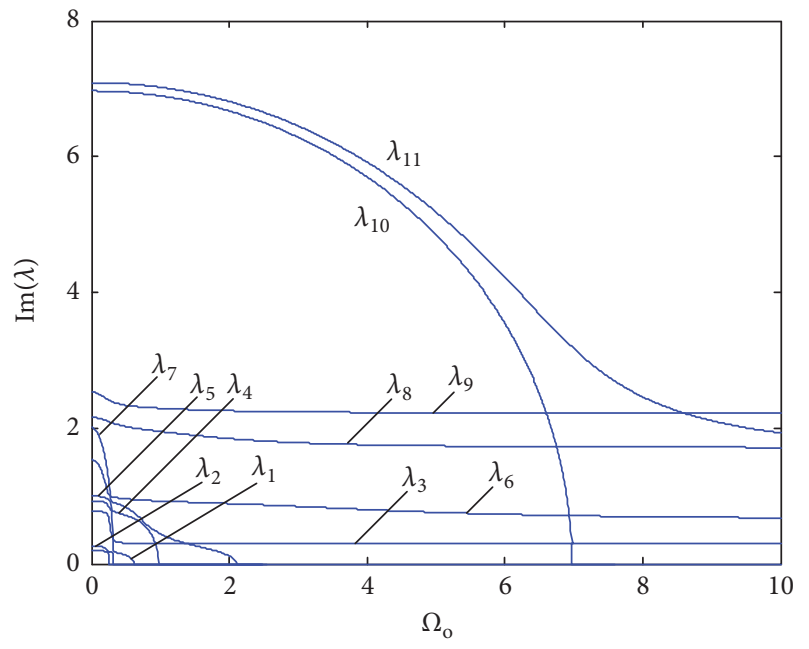

(a) Imaginary part

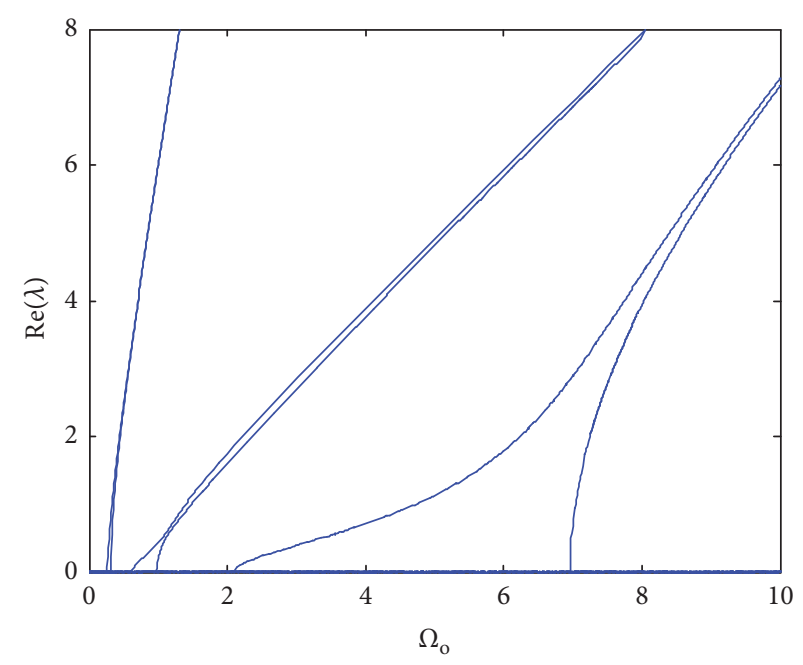

(b) Real part

FIGURE 4: Imaginary and real parts of the eigenvalue for rotational modes.

4.2. Influence Factor Analysis. The speed of the carrier is defined as critical speed $\Omega_{\text {crit }}$ where one or more of the eigenvalues are zero. The critical speed is determined by the equation as follows:

$$
\left(\mathbf{K}_{\mathrm{b}}+\mathbf{K}_{\mathrm{m}}-\Omega_{\text {crit }}^{2} \mathbf{K}_{\Omega}\right) \boldsymbol{\phi}=\mathbf{0} .
$$

Equation (16) represents an eigenvalue problem where $\Omega_{\text {crit }}^{2}$ is the eigenvalue. $\phi$ will be called the critical speed eigenvector. From the matrix symmetries, the eigenvalues $\Omega_{\text {crit }}^{2}$ and the corresponding critical speed eigenvectors $\phi$ are real-valued.

Gyroscopic systems have specific characteristics of the eigenvalues and eigenvectors. The eigenvalue for a range of nondimensional carrier speeds that includes critical speeds are calculated with parameters in Table 1 . The eigenvalue loci for three modes of RV reducer are shown in Section 4.2.1 to illustrate the unusual eigenvalue behavior.

4.2.1. Influence of Carrier Speed. Figure 4 shows the eigenvalue loci for rotational modes of $\mathrm{RV}$ reducer with three planets and four cycloid gears. Eigenvalues with $\operatorname{Im}(\lambda)<10$ are shown, while higher eigenvalues have similar behavior. Rotational modes have exactly eleven eigenvalues. All eigenvalues decrease with the speed of carrier. $\lambda_{1}, \lambda_{2}, \lambda_{4}, \lambda_{5}$, and $\lambda_{7}$ decrease rapidly to zero with carrier speed for low speed. $\lambda_{10}$ decreases slowly for low carrier speed and rapidly to zero for high carrier speed. $\lambda_{8}$ and $\lambda_{9}$ decrease slowly as carrier speed increases. $\lambda_{3}, \lambda_{6}$, and $\lambda_{11}$ decrease rapidly for low carrier speed and slowly for high speed. Rotational modes have six critical speeds at $\Omega_{\mathrm{o}}=0.7,0.32,0.63,0.99,2.11,6.97$. When the eigenvalues $\lambda_{1}, \lambda_{2}, \lambda_{4}, \lambda_{5}, \lambda_{7}$, and $\lambda_{10}$ are above critical speeds, the imaginary parts are zero and the real parts are positive. It means that the eigenvalues undergo divergence instability. For $\Omega_{0}<0.17$, all eigenvalues are purely imaginary and stable.
Figure 5 shows the eigenvalue loci for translational modes. Figure 6 shows the low-speed region indicated by the dashed box in Figure 5. The natural frequencies of translational mode are degenerate at zero carrier speed and split for nonzero carrier speed, which is typical behavior of gyroscopic system. Translational modes have twelve critical speeds. $\lambda_{1}$ decreases to zero at $\Omega_{\mathrm{o}}=0.38$, where the critical speed occurs. Stability is not lost at this speed because the eigenvalue remains purely imaginary. As the carrier speed increases from $\Omega_{\mathrm{o}}=0.38, \lambda_{1}$ increases and collides with $\lambda_{2}$ at $\Omega_{\mathrm{o}}=0.62$, where the imaginary parts and the real parts are both positive. It means that the eigenvalues experience flutter instability. For $0.62<\Omega_{0}<4.05, \lambda_{1}$ and $\lambda_{2}$ increase with the carrier speed and split at $\Omega_{\mathrm{o}}=4.05$, where the real parts of $\lambda_{1}$ and $\lambda_{2}$ vanish. The stability is recovered. Similar behavior appears to $\lambda_{11}$ and $\lambda_{12} . \lambda_{3}$ and $\lambda_{4}$ decrease rapidly to zero at $\Omega_{\mathrm{o}}=0.26$, where the critical speed occurs. For $\Omega_{\mathrm{o}}>0.26$, the eigenvalues are real and positive which means that divergence instability occurs. Hence, both flutter and divergence instability occur for translational mode.

Figure 7 shows the eigenvalue loci for planetary component modes. $\lambda_{1}$ and $\lambda_{2}$ are the eigenvalues that experience critical speeds. $\lambda_{1}$ decreased slowly for $\Omega_{\mathrm{o}}<0.21$, but it decreases rapidly for $\Omega_{\mathrm{o}}>0.21$. $\lambda_{2}$ decreases rapidly to zero. $\lambda_{3}$ decreases rapidly for $\Omega_{0}<0.23$, but it decreases slowly for $\Omega_{\mathrm{o}}>0.23$. Planetary component modes have two critical speeds at $\Omega_{\mathrm{o}}=0.32,0.33$. For $\Omega_{\mathrm{o}}>0.32$ and $\Omega_{\mathrm{o}}>0.33, \lambda_{1}$ and $\lambda_{2}$ are, respectively, real and positive which means that divergence instability occurs.

4.2.2. Influence of Crankshaft Eccentricity. To investigate the influence of crankshaft eccentricity on eigenvalue, Figure 8 shows the eigenvalue loci over a range of nondimensional carrier speeds when eccentricity is $0.9 \mathrm{~mm}$. The eigenvalues which are obviously affected are shown by dashed lines. Compared with Figure 4, the values of $\lambda_{6}$ for low speed are 


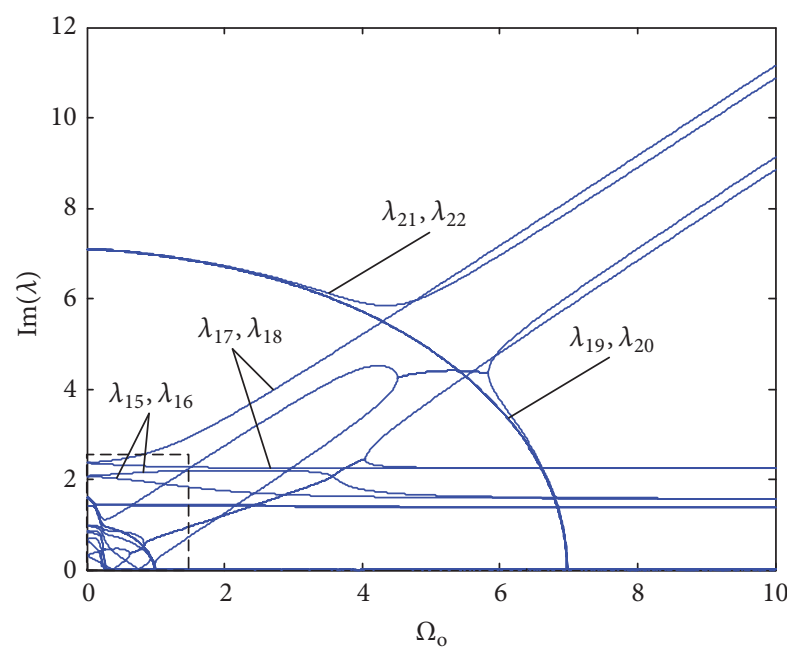

(a) Imaginary part

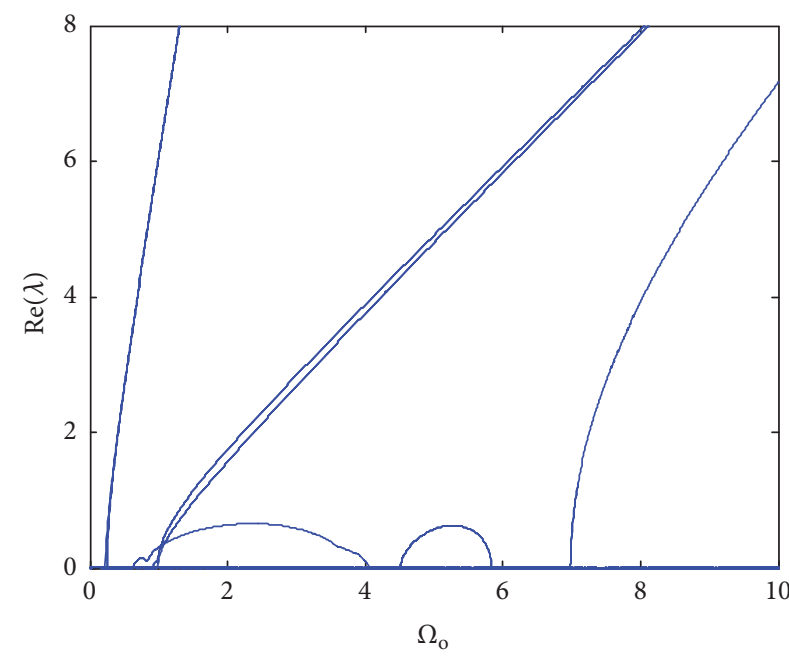

(b) Real part

FIGURE 5: Imaginary and real parts of the eigenvalue for translational modes.

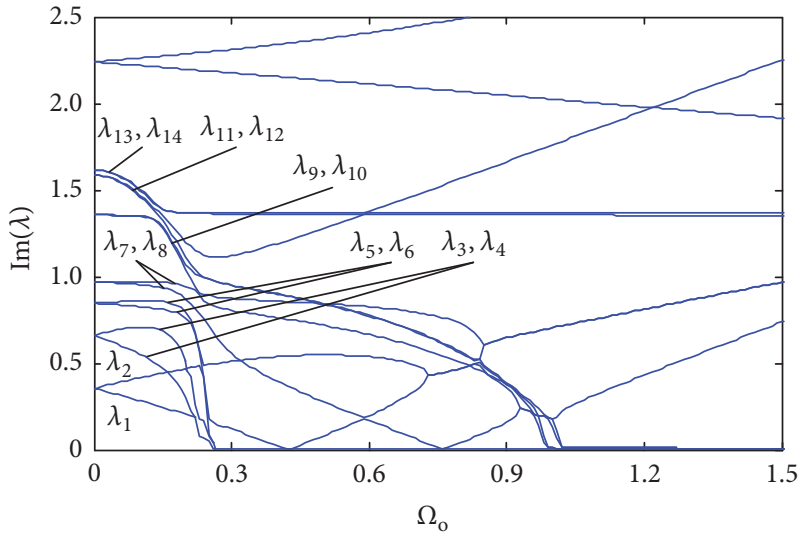

FIGURE 6: Imaginary and real parts of the eigenvalue for the range outlined in a dashed box in Figure 5.

much smaller and the overall value of $\lambda_{9}$ is smaller. Compared with Figure 5, the values of $\lambda_{15}$ and $\lambda_{16}$ associated with translational mode are smaller and the two eigenvalues have the trend to converge. However, the eigenvalues associated with planetary component mode remain unchanged. Hence, eccentricity only affects eigenvalues of rotational and translational modes.

4.2.3. Influence of Number of Pins. Figure 9 shows the eigenvalue loci with different number of pins for $0<\Omega_{\mathrm{o}}<1.5$. The eigenvalue loci with forty and forty-five pins are, respectively, shown by blue and red color. As seen in the picture, the overall trend of the eigenvalue loci remains unchanged and the number of pins mainly affects the lower eigenvalues with carrier speed for low speed. Unlike crankshaft, number of pins has influence on eigenvalues of all three type modes. The eigenvalues change a lot with large curvature of the loci.

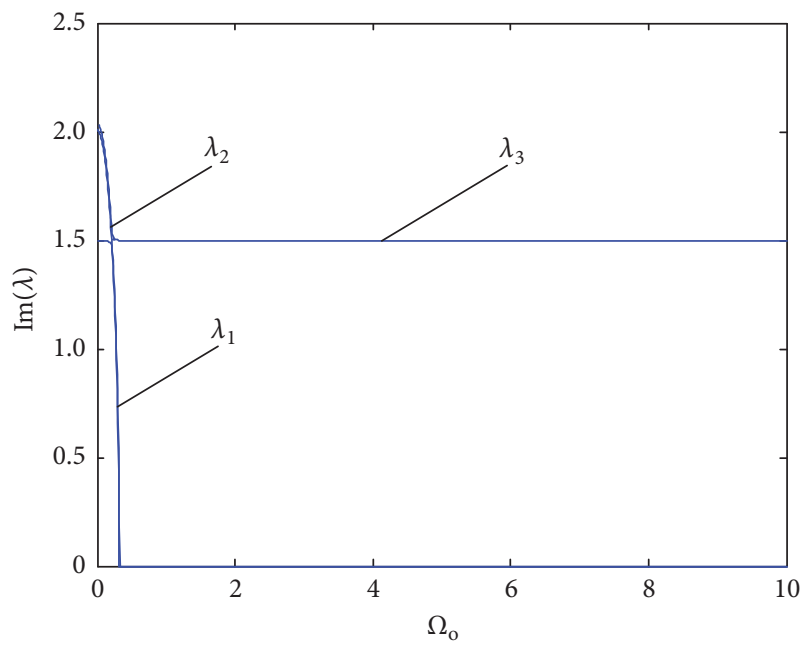

(a) Imaginary part

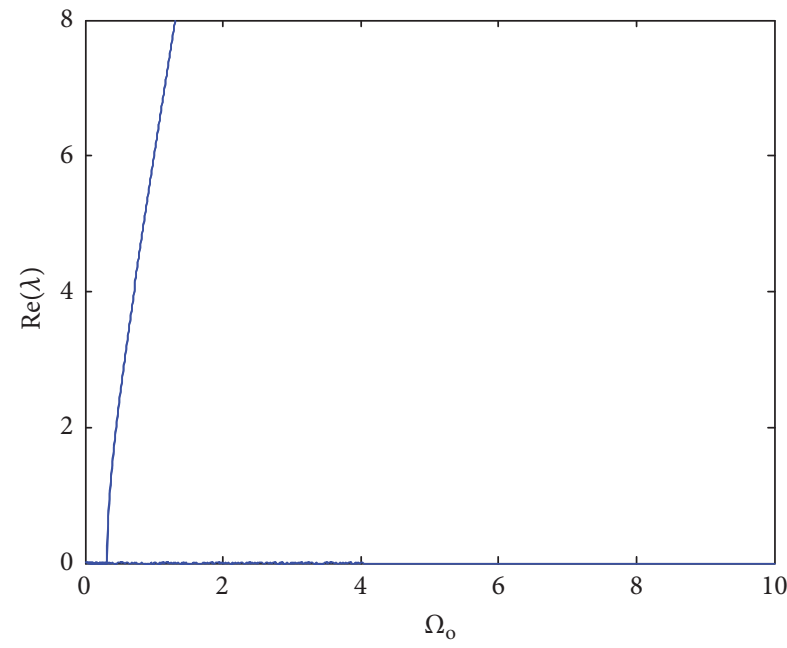

(b) Real part

FIGURE 7: Imaginary and real parts of the eigenvalue for planetary component modes. 


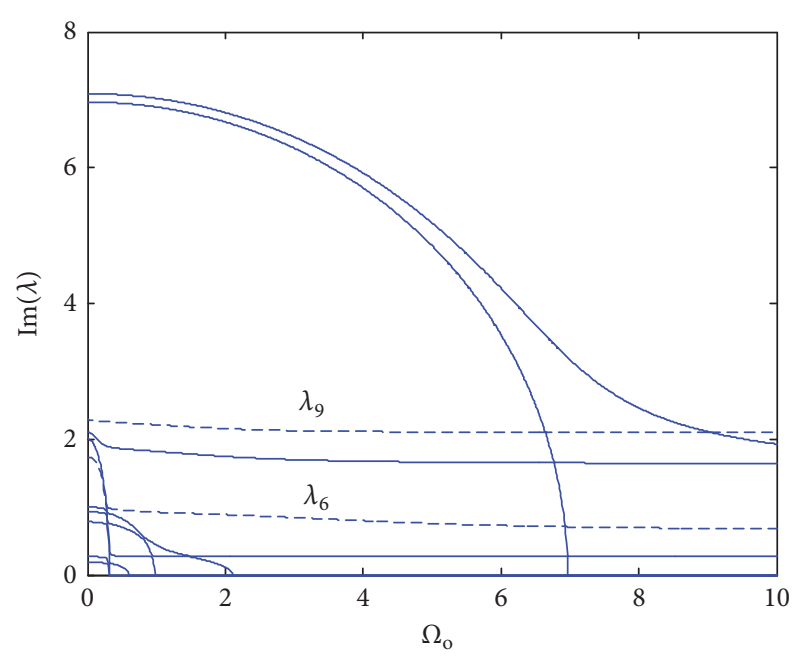

(a) Rotational mode

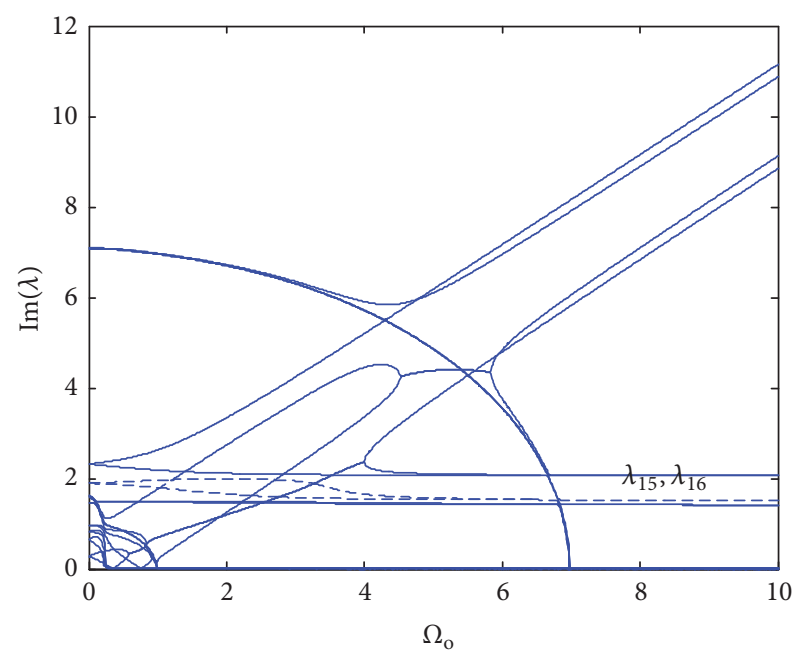

(b) Translational mode

FIGURE 8: Influence of crankshaft eccentricity.

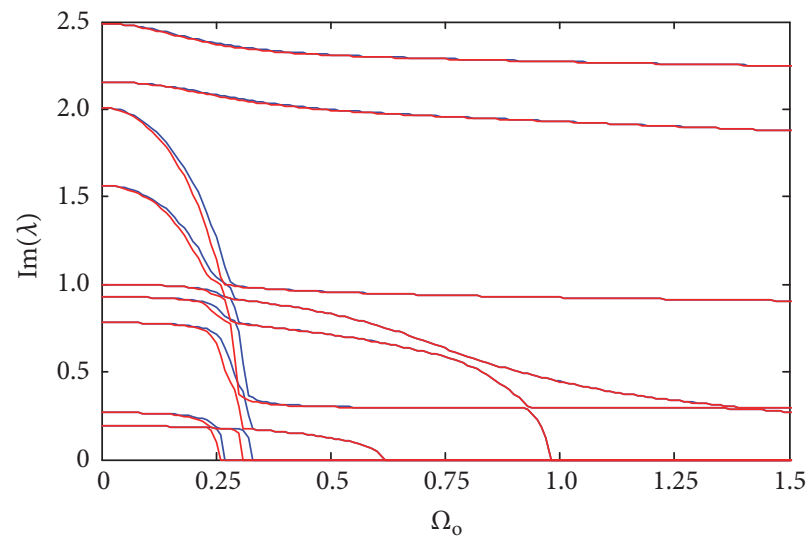

(a) Rotational mode

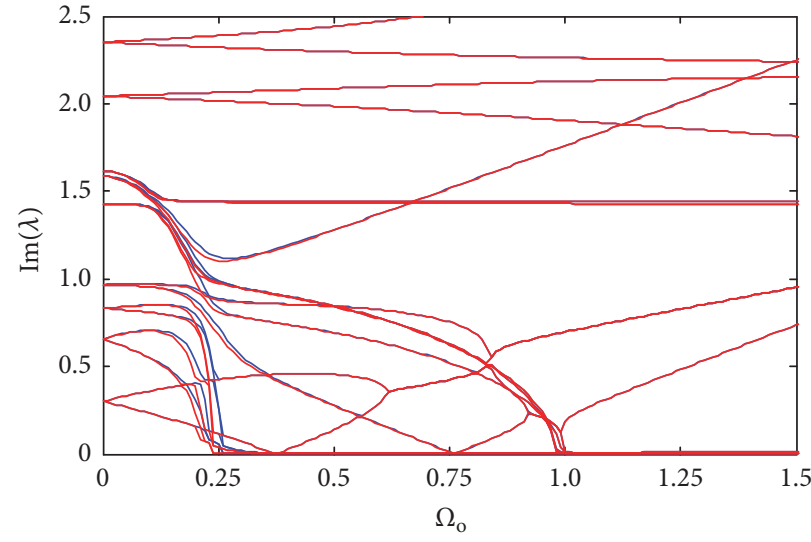

(b) Translational mode

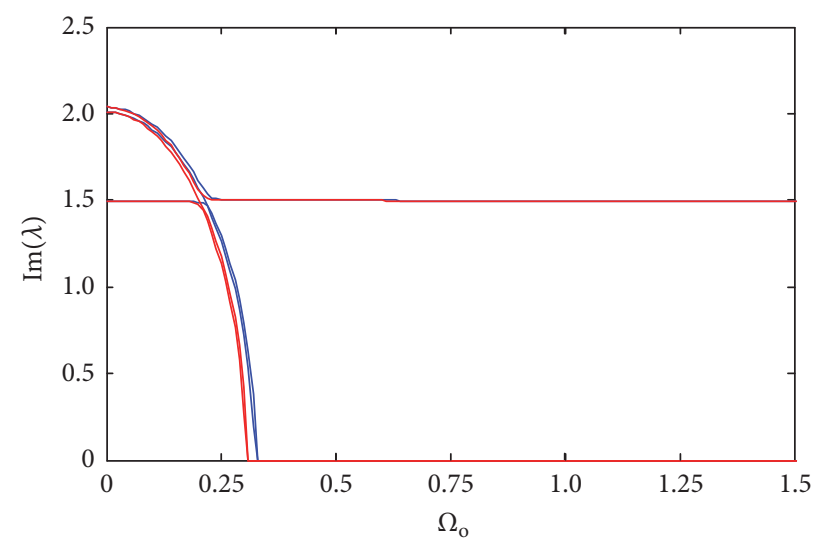

(c) Planetary mode

FIGURE 9: Influence of number of pins.

\section{Experiments}

The dynamic testing experiments are performed with engineering prototype. The basic parameters of prototype are shown in Table 4. The prototype has three planets and crankshafts and two cycloid gears. The dynamic testing setup is shown in Figure 10. The speed of the sun is controlled by the servomotor. The load is replaced with inertia plate. Vibration 
TABLE 4: Basic parameters of engineering prototype.

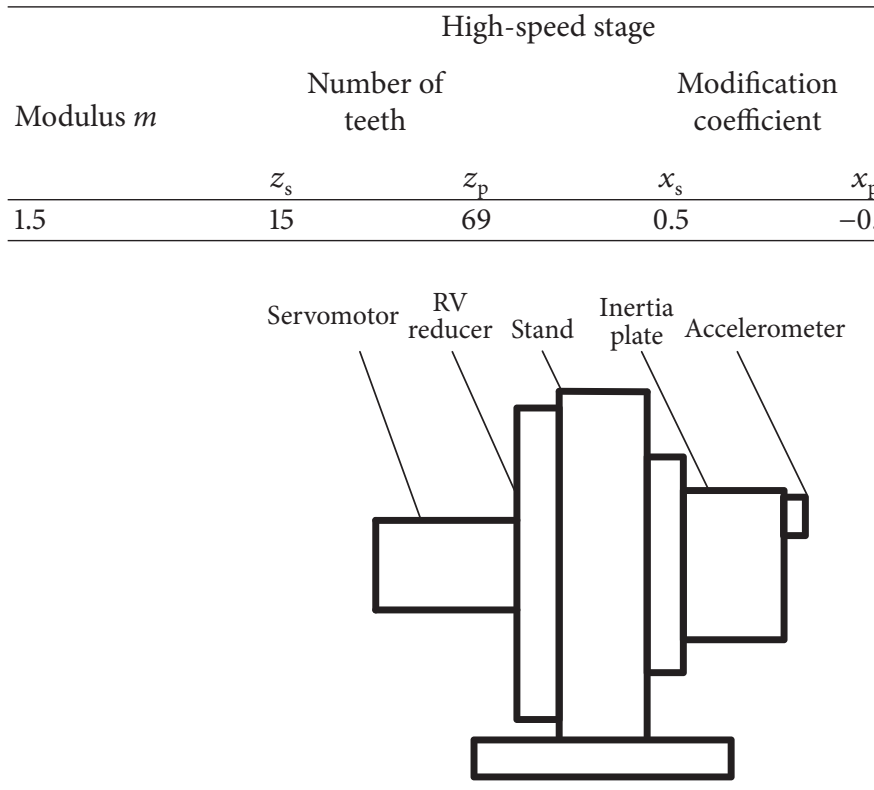

(a) Testing table

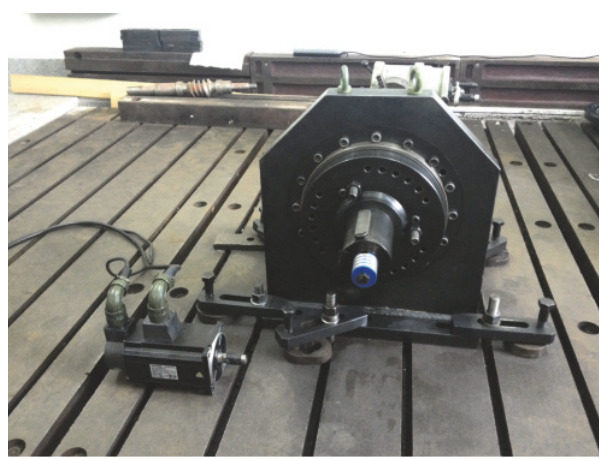

(b) Schematic of testing

FIGURE 10: RV reducer dynamic testing setup.

TABLE 5: Natural frequencies from experiment and analytical model.

\begin{tabular}{lccc}
\hline Mode Experiment $f /(\mathrm{Hz})$ & Analytical model $f /(\mathrm{Hz})$ & Error $\Delta /(\%)$ \\
\hline 1 & 148.95 & 154.56 & 3.77 \\
2 & 387.88 & 396.09 & 2.12 \\
3 & 681.47 & 697.16 & 2.30 \\
\hline
\end{tabular}

signal is collected by accelerometers mounted on the inertia plate and disposed by LMS TEST.Lab. The experimental results are listed in Table 5 and compared to analytical results calculated by (4).

Table 5 shows the first three natural frequencies obtained from dynamic testing and analytical calculation. Results of two methods are in good agreement and the maximum amplitude difference is within 5 percent, which verifies the effectiveness of the analytical dynamic model.

\section{Conclusions}

A dynamic model of general description of RV reducer has been developed to investigate free vibration characteristics and effect of key parameters on natural frequencies for gyroscopic system. The main results are the following:

(1) The natural frequencies and vibration modes have highly structured characteristics due to symmetry structure. Vibration modes for nongyroscopic and gyroscopic systems can be classified into three typical types, rotational modes, translational modes, and planetary component modes. Rotational and translational modes have pure rotation and translation of the central components. Only planetary components have motion in planetary component mode.

(2) For nongyroscopic system, the eigenvalues and eigenvectors are real. The motions of different coordinates are either in-phase or 180 deg out of phase. However, for gyroscopic system, the eigenvalues and eigenvectors are complex-valued, which physically means a phase difference exists between the motions of different coordinates.

(3) The eigenvalues of gyroscopic system are speeddependent. There are, respectively, six, twelve, and two critical speeds for rotational, translational, and planetary component modes. Divergence and flutter instability are observed at speeds adjacent to critical speeds. Crankshaft eccentricity only affects eigenvalues of rotational and translational modes.

$$
\begin{aligned}
& \mathbf{M}=\operatorname{diag}\left[\begin{array}{lllllllllll}
\mathbf{M}_{\mathrm{s}} & \mathbf{M}_{\mathrm{p} 1} & \cdots & \mathbf{M}_{\mathrm{p} M} & \mathbf{M}_{\mathrm{H} 1} & \cdots & \mathbf{M}_{\mathrm{H} M} & \mathbf{M}_{\mathrm{c} 1} & \cdots & \mathbf{M}_{\mathrm{cN}} & \mathbf{M}_{\mathrm{o}}
\end{array}\right], \\
& \mathbf{M}_{j}=\operatorname{diag}\left[\begin{array}{lll}
\mu_{j} & \mu_{j} & v_{j}
\end{array}\right] \quad(j=\mathrm{s}, \mathrm{p} 1, \ldots, \mathrm{p} M, \mathrm{H} 1, \ldots, \mathrm{H} M, \mathrm{c} 1, \ldots, \mathrm{cN}, \mathrm{o}) \text {, } \\
& \mathbf{G}=\operatorname{diag}\left[\begin{array}{lllllllllll}
\mathbf{G}_{\mathrm{s}} & \mathbf{G}_{\mathrm{p} 1} & \cdots & \mathbf{G}_{\mathrm{p} M} & \mathbf{G}_{\mathrm{H} 1} & \cdots & \mathbf{G}_{\mathrm{H} M} & \mathbf{G}_{\mathrm{c} 1} & \cdots & \mathbf{G}_{\mathrm{cN}} & \mathbf{G}_{\mathrm{o}}
\end{array}\right],
\end{aligned}
$$




$$
\begin{aligned}
& \mathbf{G}_{j}=\left[\begin{array}{ccc}
0 & -2 \mu_{j} & 0 \\
2 \mu_{j} & 0 & 0 \\
0 & 0 & 0
\end{array}\right] \quad(j=\mathrm{s}, \mathrm{p} 1, \ldots, \mathrm{p} M, \mathrm{H} 1, \ldots, \mathrm{H} M, \mathrm{c} 1, \ldots, \mathrm{cN}, \mathrm{o}),
\end{aligned}
$$

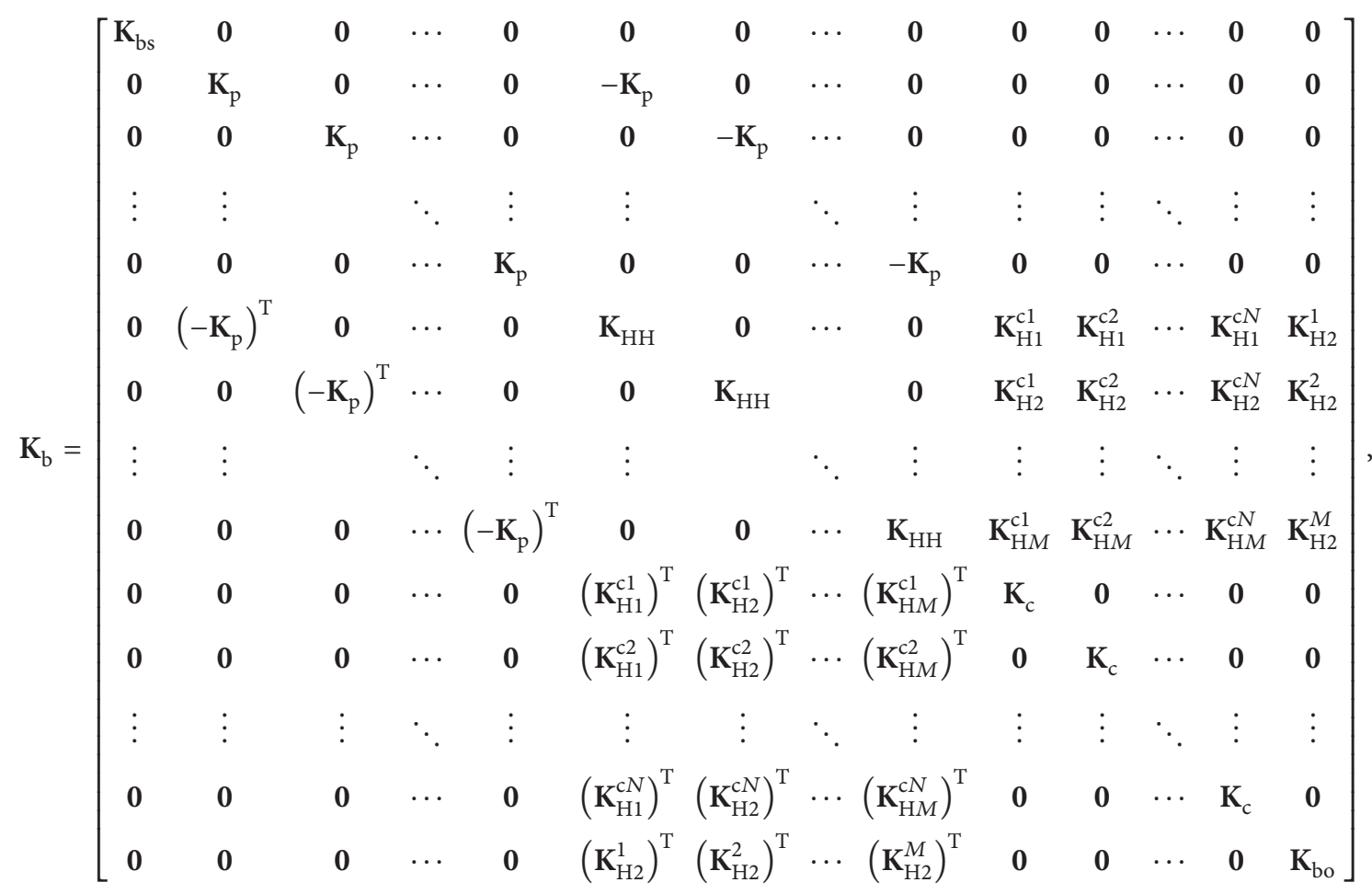

$$
\begin{aligned}
& \mathbf{K}_{\mathrm{bs}}=\operatorname{diag}\left[\begin{array}{lll}
\kappa_{\mathrm{s}} & \kappa_{\mathrm{s}} & \kappa_{\mathrm{st}}
\end{array}\right] \text {, } \\
& \mathbf{K}_{\mathrm{p}}=\operatorname{diag}\left[\begin{array}{lll}
\kappa_{\mathrm{a}} & \kappa_{\mathrm{a}} & \kappa_{\mathrm{H}}
\end{array}\right], \\
& \mathbf{K}_{\mathrm{HH}}=\mathbf{K}_{\mathrm{H} 1}+\mathbf{K}_{\mathrm{H} 2}+\mathbf{K}_{\mathrm{p}} \text {, } \\
& \mathbf{K}_{\mathrm{H} 1}=N \kappa_{\mathrm{cb}}\left[\begin{array}{ccc}
1 & 0 & 0 \\
0 & 1 & 0 \\
0 & 0 & \varepsilon
\end{array}\right] \\
& \mathbf{K}_{\mathrm{H} 2}=\kappa_{\mathrm{Hb}}\left[\begin{array}{ccc}
1 & 0 & 0 \\
0 & 1 & 0 \\
0 & 0 & 0
\end{array}\right] \\
& \mathbf{K}_{\mathrm{H} i}^{\mathrm{cj}}=\kappa_{\mathrm{cb}}\left[\begin{array}{ccc}
-\cos \psi_{\mathrm{H} i}^{\mathrm{cj}} & -\sin \psi_{\mathrm{H} i}^{\mathrm{cj}} & 0 \\
-\sin \psi_{\mathrm{H} i} & -\cos \psi_{\mathrm{H} i}^{\mathrm{cj}} & -\gamma_{\mathrm{H}} \\
0 & -\varepsilon & -\gamma_{\mathrm{H}} \varepsilon \cos \psi_{\mathrm{H} i}^{\mathrm{cj}}
\end{array}\right] \text {, } \\
& \mathbf{K}_{\mathrm{H} 2}^{i}=\kappa_{\mathrm{Hb}}\left[\begin{array}{ccc}
-\cos \psi_{\mathrm{H} i}^{\mathrm{o}} & \sin \psi_{\mathrm{H} i}^{\mathrm{o}} & 0 \\
-\sin \psi_{\mathrm{H} i}^{\mathrm{o}} & -\cos \psi_{\mathrm{H} i}^{\mathrm{o}} & -\gamma_{\mathrm{H}} \\
0 & 0 & 0
\end{array}\right] \text {, } \\
& \mathbf{K}_{\mathrm{c}}=\operatorname{diag}\left[\begin{array}{lll}
M \kappa_{\mathrm{cb}} & M \kappa_{\mathrm{cb}} & M \kappa_{\mathrm{cb}} \gamma_{\mathrm{H}}^{2}
\end{array}\right] \text {, } \\
& \mathbf{K}_{\mathrm{bo}}=\mathbf{K}_{\mathrm{bo} 1}+\mathbf{K}_{\mathrm{bo} 2} \text {, } \\
& \mathbf{K}_{\mathrm{bo} 1}=\operatorname{diag}\left[\begin{array}{lll}
\kappa_{\mathrm{o}} & \kappa_{\mathrm{o}} & \kappa_{\mathrm{ot}}
\end{array}\right] \text {, }
\end{aligned}
$$


$\mathbf{K}_{\mathrm{bo} 2}=\operatorname{diag}\left[\begin{array}{lll}M \kappa_{\mathrm{Hb}} & M \kappa_{\mathrm{Hb}} & M \kappa_{\mathrm{Hb}} \gamma_{\mathrm{H}}^{2}\end{array}\right]$,

$$
\begin{aligned}
& \mathbf{K}_{\mathrm{m}}=\left[\begin{array}{cccccccccccccc}
\sum_{i} \mathbf{K}_{s 1}^{i} & \mathbf{K}_{\mathrm{s} 2}^{1} & \mathbf{K}_{\mathrm{s} 2}^{2} & \cdots & \mathbf{K}_{\mathrm{s} 2}^{M} & \mathbf{0} & \mathbf{0} & \cdots & \mathbf{0} & \mathbf{0} & \mathbf{0} & \cdots & \mathbf{0} & \mathbf{0} \\
\left(\mathbf{K}_{\mathrm{s} 2}^{1}\right)^{\mathrm{T}} & \mathbf{K}_{\mathrm{pp}} & \mathbf{0} & \cdots & \mathbf{0} & \mathbf{0} & \mathbf{0} & \cdots & \mathbf{0} & \mathbf{0} & \mathbf{0} & \cdots & \mathbf{0} & \mathbf{0} \\
\left(\mathbf{K}_{\mathrm{s} 2}^{2}\right)^{\mathrm{T}} & \mathbf{0} & \mathbf{K}_{\mathrm{pp}} & & \mathbf{0} & \mathbf{0} & \mathbf{0} & \cdots & \mathbf{0} & \mathbf{0} & \mathbf{0} & \cdots & \mathbf{0} & \mathbf{0} \\
\vdots & \vdots & & \ddots & \vdots & \vdots & \vdots & & \vdots & \vdots & \vdots & & \vdots & \vdots \\
\left(\mathbf{K}_{\mathrm{s} 2}^{M}\right)^{\mathrm{T}} & \mathbf{0} & \mathbf{0} & \cdots & \mathbf{K}_{\mathrm{pp}} & \mathbf{0} & \mathbf{0} & \cdots & \mathbf{0} & \mathbf{0} & \mathbf{0} & \cdots & \mathbf{0} & \mathbf{0} \\
\mathbf{0} & \mathbf{0} & \mathbf{0} & \cdots & \mathbf{0} & \mathbf{0} & \mathbf{0} & \cdots & \mathbf{0} & \mathbf{0} & \mathbf{0} & \cdots & \mathbf{0} & \mathbf{0} \\
\mathbf{0} & \mathbf{0} & \mathbf{0} & \cdots & \mathbf{0} & \mathbf{0} & \mathbf{0} & \cdots & \mathbf{0} & \mathbf{0} & \mathbf{0} & \cdots & \mathbf{0} & \mathbf{0} \\
\vdots & \vdots & \vdots & \ddots & \vdots & \vdots & \vdots & \ddots & \vdots & \vdots & \vdots & \ddots & \vdots & \vdots \\
\mathbf{0} & \mathbf{0} & \mathbf{0} & \cdots & \mathbf{0} & \mathbf{0} & \mathbf{0} & \cdots & \mathbf{0} & \mathbf{0} & \mathbf{0} & \cdots & \mathbf{0} & \mathbf{0} \\
\mathbf{0} & \mathbf{0} & \mathbf{0} & \cdots & \mathbf{0} & \mathbf{0} & \mathbf{0} & \cdots & \mathbf{0} & \mathbf{K}_{\mathrm{cr}} & \mathbf{0} & \cdots & \mathbf{0} & \mathbf{0} \\
\mathbf{0} & \mathbf{0} & \mathbf{0} & \cdots & \mathbf{0} & \mathbf{0} & \mathbf{0} & \cdots & \mathbf{0} & \mathbf{0} & \mathbf{K}_{\mathrm{cr}} & & \mathbf{0} & \mathbf{0} \\
\vdots & \vdots & \vdots & \ddots & \vdots & \vdots & \vdots & \ddots & \vdots & & & \ddots & & \\
\mathbf{0} & \mathbf{0} & \mathbf{0} & \cdots & \mathbf{0} & \mathbf{0} & \mathbf{0} & \cdots & \mathbf{0} & \mathbf{0} & \mathbf{0} & \cdots & \mathbf{K}_{\mathrm{cr}} & \mathbf{0} \\
\mathbf{0} & \mathbf{0} & \mathbf{0} & \cdots & \mathbf{0} & \mathbf{0} & \mathbf{0} & \cdots & \mathbf{0} & \mathbf{0} & \mathbf{0} & \cdots & \mathbf{0} & \mathbf{0}
\end{array}\right] \\
& \mathbf{K}_{\mathrm{s} 1}^{i}=\kappa_{\mathrm{si}}\left[\begin{array}{ccc}
\cos ^{2} \psi_{\mathrm{si}} & \cos \psi_{\mathrm{si}} \sin \psi_{\mathrm{si}} & \gamma_{\mathrm{s}} \cos \psi_{\mathrm{si}} \\
\cos \psi_{\mathrm{si}} \sin \psi_{\mathrm{si}} & \sin ^{2} \psi_{\mathrm{si}} & \gamma_{\mathrm{s}} \sin \psi_{\mathrm{si}} \\
\gamma_{\mathrm{s}} \cos \psi_{\mathrm{si}} & \gamma_{\mathrm{s}} \sin \psi_{\mathrm{si}} & \gamma_{\mathrm{s}}^{2}
\end{array}\right] \text {, } \\
& \mathbf{K}_{\mathrm{s} 2}^{i}=\kappa_{\mathrm{s} i}\left[\begin{array}{ccc}
-\cos \psi_{\mathrm{si}} \sin \alpha_{\mathrm{s}} & -\cos \psi_{\mathrm{si}} \cos \alpha_{\mathrm{s}} & \gamma_{\mathrm{p}} \cos \psi_{\mathrm{s} i} \\
-\sin \psi_{\mathrm{si}} \sin \alpha_{\mathrm{s}} & -\sin \psi_{\mathrm{si}} \cos \alpha_{\mathrm{s}} & \gamma_{\mathrm{p}} \sin \psi_{\mathrm{si}} \\
-\gamma_{\mathrm{s}} \sin \alpha_{\mathrm{s}} & -\gamma_{\mathrm{s}} \cos \alpha_{\mathrm{s}} & \gamma_{\mathrm{s}} \gamma_{\mathrm{p}}
\end{array}\right] \text {, } \\
& \mathbf{K}_{\mathrm{pp}}=\kappa_{\mathrm{si}}\left[\begin{array}{ccc}
\sin ^{2} \alpha_{\mathrm{s}} & \cos \alpha_{\mathrm{s}} \sin \alpha_{\mathrm{s}} & -\gamma_{\mathrm{p}} \sin \alpha_{\mathrm{s}} \\
\sin \alpha_{\mathrm{s}} \cos \alpha_{\mathrm{s}} & \cos ^{2} \alpha_{\mathrm{s}} & -\gamma_{\mathrm{p}} \cos \alpha_{\mathrm{s}} \\
-\gamma_{\mathrm{p}} \sin \alpha_{\mathrm{s}} & -\gamma_{\mathrm{p}} \cos \alpha_{\mathrm{s}} & \gamma_{\mathrm{p}}^{2}
\end{array}\right] \text {, } \\
& \mathbf{K}_{\mathrm{cr}}=\kappa_{\mathrm{cr}}\left[\begin{array}{ccc}
\sin ^{2} \beta & \sin \beta \cos \beta & \gamma_{\mathrm{c}} \cos \beta \sin \beta \\
\sin \beta \cos \beta & \cos ^{2} \beta & \gamma_{\mathrm{c}} \cos ^{2} \beta \\
\gamma_{\mathrm{c}} \cos \beta \sin \beta & \gamma_{\mathrm{c}} \cos ^{2} \beta & \gamma_{\mathrm{c}}^{2} \cos ^{2} \beta
\end{array}\right] \text {. }
\end{aligned}
$$

\section{Conflicts of Interest}

The authors declare that they have no conflicts of interest.

\section{Acknowledgments}

The work is based upon work supported by the National High Technology Research and Development Program of China (863 Program) (no. 2011AA04A102).

\section{References}

[1] M. Botman, "Epicyclic gear vibrations," Journal of Engineering for Industry, vol. 98, no. 3, pp. 811-815, 1976.

[2] A. Kahraman, "Natural modes of planetary gear trains," Journal of Sound and Vibration, vol. 173, no. 1, pp. 125-130, 1994.

[3] J. Lin and R. G. Parker, "Analytical characterization of the unique properties of planetary gear free vibration," Journal of Vibration and Acoustics, vol. 121, no. 3, pp. 316-321, 1999. 
[4] A. Kahraman, "Free torsional vibration characteristics of compound planetary gear sets," Mechanism and Machine Theory, vol. 36, no. 8, pp. 953-971, 2001.

[5] D. R. Kiracofe and R. G. Parker, "Structured vibration modes of general compound planetary gear systems," Journal of Vibration and Acoustics, vol. 129, no. 1, pp. 1-16, 2007.

[6] Y. Guo and R. G. Parker, "Purely rotational model and vibration modes of compound planetary gears," Mechanism and Machine Theory, vol. 45, no. 3, pp. 365-377, 2010.

[7] J. Lin and R. G. Parker, "Natural frequency veering in planetary gears," Mechanics of Structures and Machines, vol. 29, no. 4, pp. 411-429, 2001.

[8] T. M. Ericson and R. G. Parker, "Natural frequency clusters in planetary gear vibration," Journal of Vibration and Acoustics, vol. 135, no. 6, Article ID 061002, 2013.

[9] M. Blagojevic, V. Nikolic-Stanojevic, N. Marjanovic et al., "Analysis of cycloid drive dynamic behavior," Scientific Technical Review, vol. 59, no. 1, p. 52, 2009.

[10] L. Pascale, M. Neagoe, D. Diaconescu et al., "The dynamic modeling of a new cycloidal planetary gear pair with rollers used in robots orientation system," The Scientific Bulletin of Electrical Engineering Faculty, vol. 1, p. 10, 2009.

[11] Y.-H. Zhang, J.-J. Xiao, and W.-D. He, "Dynamical formulation and analysis of RV reducer," in Proceedings of the International Conference on Engineering Computation (ICEC '09), pp. 201204, IEEE, Hong Kong, May 2009.

[12] Y. Zhang, W. He, and J. Xiao, "Dynamical model of RV reducer and key influence of stiffness to the nature character," in Proceedings of the 3rd International Conference on Information and Computing (ICIC '10), pp. 192-195, Chengdu, China, June 2010.

[13] C.-F. Hsieh, "Dynamics analysis of cycloidal speed reducers with pinwheel and nonpinwheel designs," Journal of Mechanical Design, Transactions of the ASME, vol. 136, no. 9, Article ID 091008, 2014.

[14] C. Chen and Y. Yang, "Free vibration properties of rotate vector reducer," Journal of Vibroengineering, vol. 18, no. 5, pp. 30893103, 2016.

[15] J. A. Wickert and C. D. Mote Jr., "Classical vibration analysis of axially moving continua," Journal of Applied Mechanics, Transactions ASME, vol. 57, no. 3, pp. 738-744, 1990.

[16] R. G. Parker, "Supercritical speed stability of the trivial equilibrium of an axially-moving string on an elastic foundation," Journal of Sound and Vibration, vol. 221, no. 2, pp. 205-219, 1999.

[17] R. P. S. Han and J. W.-Z. Zu, "Modal analysis of rotating shafts: a body-fixed axis formulation approach," Journal of Sound and Vibration, vol. 156, no. 1, pp. 1-16, 1992.

[18] S. A. Tobias and R. N. Arnold, "The influence of dynamical imperfection on the vibration of rotating disks," Proceedings of the Institution of Mechanical Engineers, vol. 171, no. 1957, pp. 669-690, 1957.

[19] W. D. Iwan and T. L. Moeller, "The stability of a spinning elastic disk with a transverse load system," Journal of Applied Mechanics, vol. 43, no. 3, pp. 485-490, 1976.

[20] J.-S. Chen and D. B. Bogy, "Effects of load parameters on the natural frequencies and stability of a flexible spinning disk with a stationary load system," Transactions of the ASME. Journal of Applied Mechanics, vol. 59, no. 2, part 2, pp. 5230-5235, 1992.

[21] R. G. Parker and P. J. Sathe, "Free vibration and stability of a spinning disk-spindle system," Journal of Vibration and Acoustics, Transactions of the ASME, vol. 121, no. 3, pp. 391-396, 1999.
[22] R. G. Parker and P. J. Sathe, "Exact solutions for the free and forced vibration of a rotating disk-spindle system," Journal of Sound and Vibration, vol. 223, no. 3, pp. 445-465, 1999.

[23] Y. Wang, J. Yang, X. Li, G. Sun, and T. Lim, "Interaction of gear-shaft dynamics considering gyroscopic effect of compliant driveline system," SAE International Journal of Passenger Cars-Mechanical Systems, vol. 8, no. 2, pp. 742-747, 2015.

[24] C. D’Angelo III and C. D. Mote Jr., "Natural frequencies of a thin disk, clamped by thick collars with friction at the contacting surfaces, spinning at high rotation speed," Journal of Sound and Vibration, vol. 168, no. 1, pp. 1-14, 1993.

[25] J. Lin and R. G. Parker, "Sensitivity of planetary gear natural frequencies and vibration modes to model parameters," Journal of Sound and Vibration, vol. 228, no. 1, pp. 109-128, 1999.

[26] V. Abousleiman, P. Velex, and S. Becquerelle, "Modeling of spur and helical gear planetary drives with flexible ring gears and planet carriers," Journal of Mechanical Design, vol. 129, no. 1, pp. 95-106, 2007.

[27] C. G. Cooley and R. G. Parker, "Vibration properties of high-speed planetary gears with gyroscopic effects," Journal of Vibration and Acoustics, vol. 134, no. 6, Article ID 061014, 2012.

[28] C. G. Cooley and R. G. Parker, "Unusual gyroscopic system eigenvalue behavior in high-speed planetary gears," Journal of Sound and Vibration, vol. 332, no. 7, pp. 1820-1828, 2013.

[29] C. G. Cooley and R. G. Parker, "Mechanical stability of highspeed planetary gears," International Journal of Mechanical Sciences, vol. 69, no. 4, pp. 59-71, 2013. 


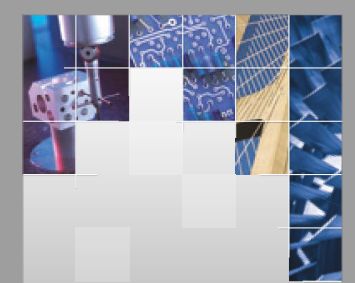

\section{Enfincering}
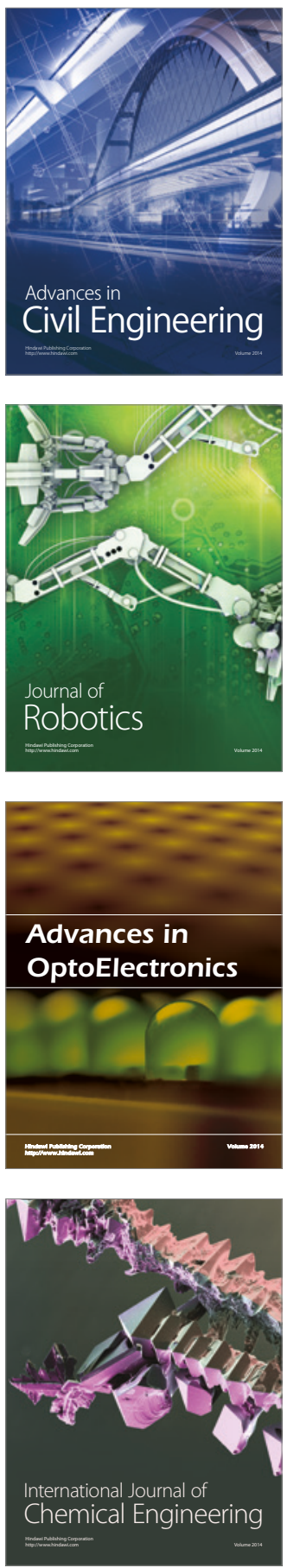

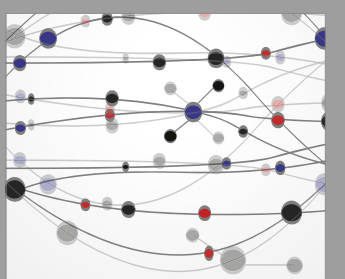

The Scientific World Journal

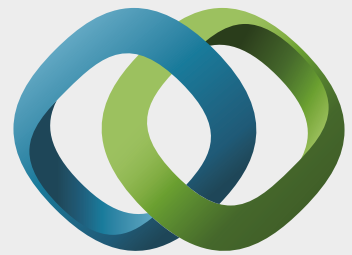

\section{Hindawi}

Submit your manuscripts at

https://www.hindawi.com
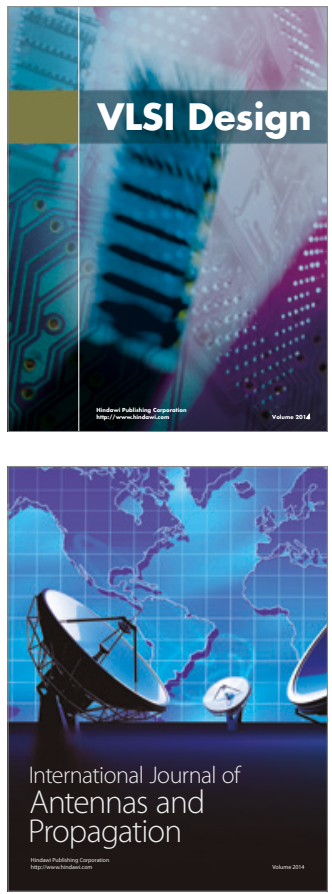

\section{Rotating}

Machinery
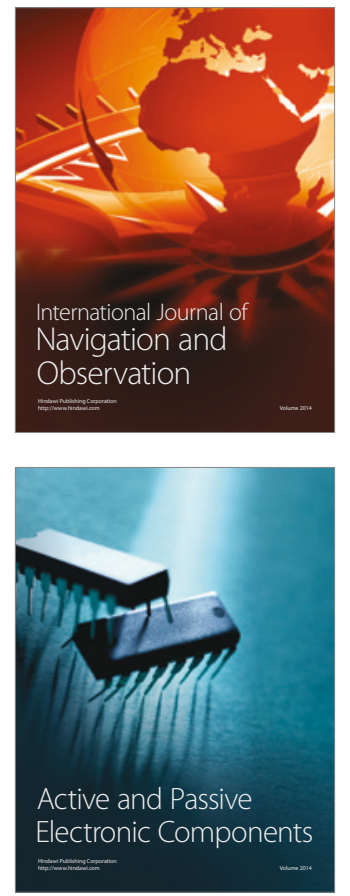
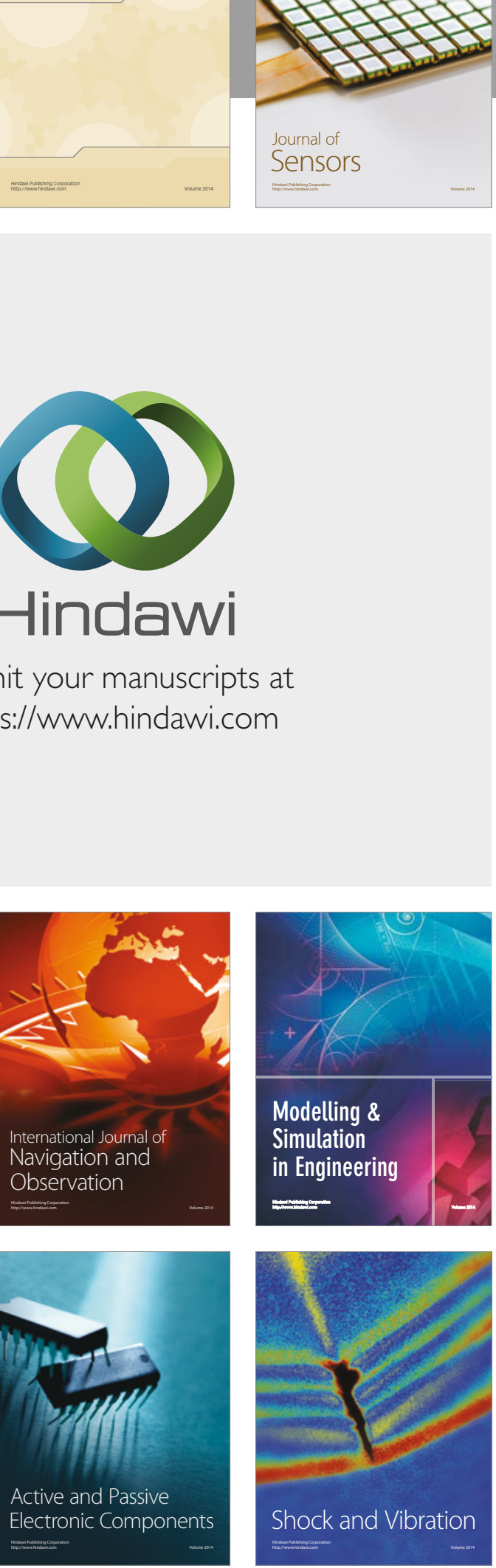
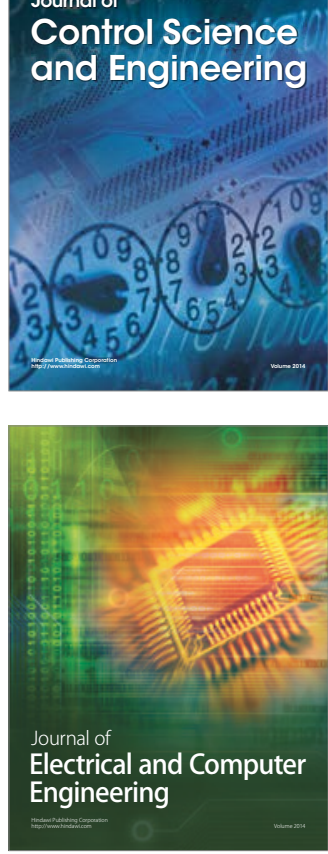

Distributed

Journal of

Control Science

and Engineering
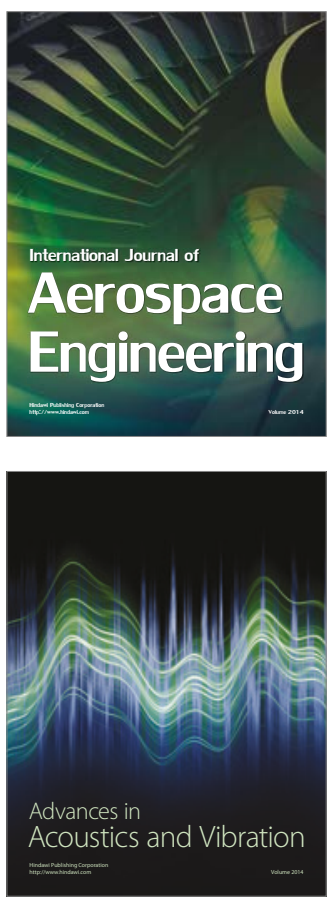

Sensor Networks 